\title{
eEF3 promotes late stages of tRNA translocation on the ribosome
}

Namit Ranjan ${ }^{1,6, *}$, Agnieszka A. Pochopien ${ }^{2,3,6}$, Colin Chih-Chien $\mathrm{Wu}^{4,6}$, Bertrand Beckert $^{3}$, Sandra Blanchet ${ }^{1}$, Rachel Green ${ }^{4,5,{ }^{*}}$, Marina V. Rodnina ${ }^{1, *}$, Daniel N. Wilson ${ }^{2,3,7,{ }^{*}}$

${ }^{1}$ Department of Physical Biochemistry, Max Planck Institute for Biophysical Chemistry, Am Fassberg 11, 37077 Göttingen, Germany.

${ }^{2}$ Gene Center, Department for Biochemistry and Center for integrated Protein Science Munich (CiPSM), University of Munich, Feodor-Lynenstr. 25, 81377 Munich, Germany

${ }^{3}$ Institute for Biochemistry and Molecular Biology, University of Hamburg, MartinLuther-King-Platz 6, 20146 Hamburg, Germany

${ }^{4}$ Department of Molecular Biology and Genetics, Johns Hopkins University School of Medicine, MD21205 Baltimore, United States.

${ }^{5}$ Howard Hughes Medical Institute, Johns Hopkins University School of Medicine, MD21205 Baltimore, United States.

${ }^{6}$ These authors contributed equally

*Correspondence: daniel.wilson@chemie.uni-hamburg.de, rodnina@mpibpc.mpg.de, ragreen@jhmi.edu, namit.ranjan@mpibpc.mpg.de 


\section{Summary}

In addition to the conserved translation elongation factors eEF1A and eEF2, fungi require a third essential elongation factor, eEF3. While eEF3 has been implicated in tRNA binding and release at the $\mathrm{A}$ and $\mathrm{E}$ sites, its exact mechanism of action is unclear. Here we show that eEF3 acts at the mRNA-tRNA translocation step by promoting the dissociation of the tRNA from the E site, but independent of aminoacyl-tRNA recruitment to the A site. Depletion of eEF3 in vivo leads to a general slow-down in translation elongation due to accumulation of ribosomes with an occupied A site. Cryo-EM analysis of ex vivo eEF3ribosome complexes shows that eEF3 facilitates late steps of translocation by favoring nonrotated ribosomal states as well as by opening the L1 stalk to release the E-site tRNA. Additionally, our analysis provides structural insights into novel translation elongation states, enabling presentation of a revised yeast translation elongation cycle.

Keywords: ABC ATPase, cryo-EM, eEF3, E-site tRNA, L1 stalk, translation elongation, translocation, yeast. 


\section{Introduction}

Protein synthesis is an evolutionary conserved process. Translation elongation entails the same repetitive steps of decoding, peptide bond formation, and translocation in all living organisms, catalyzed by highly homologous translation elongation factors eEF1A/EF-Tu and eEF2/EF-G in eukaryotes and bacteria, respectively, assisted by eEF1B/EF-Ts and eIF5A/EF-P (Dever and Green, 2012; Rodnina, 2018). It is therefore particularly surprising that some fungi, such as Saccharomyces cerevisiae, Candida albicans and Pneumocystis carinii have an additional essential, abundant elongation factor, eEF3 (Colthurst et al., 1991; Kamath and Chakraburtty, 1989; Skogerson and Wakatama, 1976; Uritani and Miyazaki, 1988). Moreover, recent bioinformatic analyses indicate that eEF3-like proteins exist more broadly in unicellular eukaryotes, not just in fungi (Mateyak et al., 2018). Some organisms, e.g. S. cerevisiae, also contains an eEF3 homologue, termed New1p, which when overexpressed can rescue strains depleted for eEF3 (Kasari et al., 2019b). In exponentially growing $S$. cerevisiae cells, eEF3, eEF1A, eEF2, and eRF1 exert the strongest control over the rate of translation (Firczuk et al., 2013). In yeast, the amount of eEF3 per cell is comparable to that of ribosomes, eEF2, and eEF1B, 10 times lower than that of eEF1A, and 5-10 times higher than of the termination factors and the majority of initiation factors (Firczuk et al., 2013). These data suggest that there is sufficient eEF3 to participate in every round of translation elongation.

eEF3 is a cytoplasmic protein that belongs to the superfamily of ATP-binding cassette (ABC) proteins, specifically, of the F subtype (Murina et al., 2018). In $S$. cerevisiae, eEF3 consists of a single polypeptide chain of 1044 amino acids comprising several domains: a HEAT-repeat domain, a four-helix bundle domain (4HB domain), two ATP-binding domains, $\mathrm{ABC} 1$ and $\mathrm{ABC} 2$, with a chromodomain (CD) insertion within ABC2, as well as eEF3-specific C-terminal domain (Kambampati et al., 2000; Murina et al., 2018). A low-resolution (9.9 $\AA$ ) cryo-electron microscopy (cryo-EM) structure of the ribosome-eEF3 complex revealed that eEF3 binds to the ribosome, spanning across the boundary between the head of the $40 \mathrm{~S}$ subunit and the central protuberance of the $60 \mathrm{~S}$ subunit, where it contacts ribosomal RNA (rRNA) and proteins, but should not interfere with the binding of eEF1A or eEF2 (Andersen et al., 2006). Despite these structural clues, the function of eEF3 remains poorly understood. Early studies reported that eEF3 facilitates 
binding of the ternary complex eEF1A-GTP-aminoacyl-tRNA to the decoding site (A site) of the ribosome, thus increasing the fidelity of translation (Uritani and Miyazaki, 1988); genetic and physical interactions between eEF1A and eEF3 appear to support this hypothesis (Anand et al., 2006; Anand et al., 2003). An in vitro study of tRNA binding to the ribosome argued that eEF3 accelerates dissociation of tRNA from the exit site (E site) independent of translocation, and is required for aminoacyl-tRNA binding to the A site when the E site is occupied (Triana-Alonso et al., 1995); thus, according to this model, eEF3 regulates decoding. By contrast, another study proposed that eEF3 facilitates the release of tRNA from the $\mathrm{P}$ site during the disassembly of post-termination complexes at the ribosome recycling step of translation (Kurata et al., 2013).

eEF3 has also been implicated in translational control in response to environmental stress. Expression of eEF3 is altered during morphogenesis in Aspergillus fumigatus and upon starvation in S. cerevisiae (Grousl et al., 2013; Kubitschek-Barreira et al., 2013). Interaction of eEF3 with the ribosome in $S$. cerevisiae is affected by $\mathrm{Stm} 1$, a protein that binds to the mRNA entry tunnel of the ribosome (Ben-Shem et al., 2011), inhibits translation at the onset of the elongation cycle and promotes decapping of a subclass of mRNAs (Balagopal and Parker, 2011; Van Dyke et al., 2009). In yeast lacking Stm1, eEF3 binding to ribosomes is enhanced and overexpression of eEF3 impairs growth (Van Dyke et al., 2009). On the other hand, eEF3 affects the function of Gcn1, thereby modulating the kinase activity of Gcn2 (Sattlegger and Hinnebusch, 2005). In addition to these functional interactions, the Saccharomyces genome and the STRING databases suggest that eEF3 interacts with a number of proteins that may affect the efficiency of translation (Cherry et al., 2012; Szklarczyk et al., 2019), including eEF2, heat shock proteins, or She2 and She3, the adaptor proteins that facilitate targeted transport of distinct mRNAs (Heym and Niessing, 2012). Together these data suggest that eEF3 may act as a hub for translational control, but insight into the role that eEF3 plays within these regulatory networks is hampered by the lack of understanding of its basic function.

Here, we address the function of eEF3 using a combination of rapid kinetics in a fully reconstituted yeast in vitro translation system, ribosome profiling, and cryo-EM. First, we show using biochemical and kinetic approaches that eEF3 helps eEF2 to complete tRNA-mRNA translocation by accelerating the late stages of the process. While eEF3 
facilitates E-site tRNA release, this activity is not dependent on the binding of ternary complex to the A site, as previously reported (Triana-Alonso et al., 1995). Ribosome profiling of strains conditionally depleted of eEF3 reveals global changes in ribosome state in the cell wherein ribosomes accumulate with an occupied A site (typical of ribosomes in a pre-translocation state). These data are consistent with the presence of a new rate-limiting step in elongation where ribosomes cannot undergo translocation due to the presence of deacylated tRNA in the E site. Cryo-EM of ex-vivo eEF3-ribosome complexes provides a structural basis for the mechanism of action of eEF3 to promote late steps of translocation as well as facilitate E-site tRNA release. 


\section{Results}

\section{eEF3 promotes late steps of tRNA-mRNA translocation}

To understand the function of eEF3, we used a fully reconstituted translation system from yeast components (Figure S1A). We assembled an 80S initiation complex (80S IC) using purified $40 \mathrm{~S}$ and $60 \mathrm{~S}$ subunits, mRNA and initiator tRNA $\left(\left[{ }^{3} \mathrm{H}\right] \mathrm{Met}-\mathrm{tRNA}_{i}{ }^{\text {Met }}\right)$ in the presence of initiation factors eIF1, eIF1A, eIF2, eIF3, eIF5, and eIF5B (Figure S1B) (STAR Methods). Addition of $\left[{ }^{14} \mathrm{C}\right] \mathrm{Phe}-\mathrm{tRNA}{ }^{\text {Phe }}$ in the ternary complex with eEF1A and GTP (TC-Phe), and eIF5A resulted in decoding of the next codon UUU and formation of the dipeptide MetPhe (Figure 1A). To monitor the dipeptide formation in real time, we carried out experiments in a quench-flow apparatus, isolated dipeptides by HPLC and quantified the extent of dipeptide formation by radioactivity counting. The rate of MetPhe formation $\left(0.15 \mathrm{~s}^{-1}\right)$ was not affected by the addition of saturating amounts of eEF3 (Figure 1B and S2A). Similarly, formation of the MetVal peptide measured on an mRNA with Val (GUU) codon was not affected by the presence of eEF3 (Figure S2B). These results indicate that eEF3 is not essential for the first round of decoding or peptide bond formation. By contrast, when we prepared an $80 \mathrm{~S}$ complex carrying a dipeptidyl-tRNA (MetPhe) in the A site in a pre-translocation state (denoted as $80 \mathrm{~S} 2 \mathrm{C}$ for dipeptide complex) and added a TC with Val-tRNA ${ }^{\mathrm{Val}}$ reading the GUU codon, the tripeptide MetPheVal was formed only when both eEF2 and eEF3 were added together with TC-Val (Figure 1C). The effect was less dramatic when Phe and Val codons were reverted, as some MetValPhe was also formed in the absence of eEF3, but the addition of eEF3 increased the rate of tripeptide formation by 10-fold, from $0.03 \mathrm{~s}^{-1}$ to $0.3 \mathrm{~s}^{-1}$ (Figure S2C).

To form tripeptide, the ribosome has to move along the mRNA in a step referred to as translocation and then bind the next aminoacyl-tRNA (Figure 1A). Either of these steps could require the help of eEF3. To identify the exact timing of eEF3 action during the elongation cycle, we incubated $80 \mathrm{~S} 2 \mathrm{C}$ with eEF2 or eEF3 alone, or with both factors together, for $15 \mathrm{~min}$ and then added TC-Val (Figure 1D). In this case, the rate of tripeptide formation was very similar and high with eEF2, or eEF2 and eEF3, about $0.4 \mathrm{~s}^{-1}$. This indicates that (i) eEF3 is not required for the decoding of the Val codon or peptide bond formation per se, and (ii) eEF2 alone can complete the translocation process given enough time. However, to function within physiological timeframes, the reaction requires eEF3. 
Interestingly, eEF3 alone facilitated translocation, albeit only on a fraction of ribosomes, as seen by the endpoint defect (Figure 1D). We speculate that eEF3 on its own promoted tRNA translocation, albeit not to the full extent.

We next specifically studied the role of eEF3 in translocation. Translocation is a dynamic process that entails several steps. Analogous to the well-studied bacterial translocation process (Belardinelli et al., 2016; Zhou et al., 2014), translocation in yeast likely includes the following major steps: (1) Prior to eEF2 binding, the tRNAs move into hybrid states upon peptidyl transfer, with concomitant rotation of the ribosomal subunits. (2) eEF2 binds and catalyzes movement of tRNAs into the so-called chimeric states, with displacement of the mRNA together with tRNA anticodons on the small subunit and accommodation of the CCA-3' end of the peptidyl-tRNA in the P site (Figure 2A). (3) mRNA and the two tRNAs move to the E and P sites of the small subunit, the small subunit rotates backward relative to the large subunit, and the eEF2 and the E-site tRNA dissociate from the ribosome (Figure 2A). One diagnostic test for the completion of step (2) is the extent of the reaction of peptidyl-tRNA with the antibiotic puromycin (Pmn). In the absence of translocation (the $80 \mathrm{~S} 2 \mathrm{C}$ complex), peptidyl-tRNA does not react with Pmn, even with very long incubation times (Figure 2B, brown triangles). Upon addition of eEF2 and eEF3, or eEF2 alone, peptidyl-tRNA reacts with Pmn. The rate of reaction with puromycin is indistinguishable from that observed for the post-translocation complex prepared by prolonged pre-incubation with the factors (Figure S2D-F), indicating that eEF2 alone is sufficient to facilitate the early stages of translocation through step (2). We note that in the presence of eEF3 and eEF2, the reaction with Pmn was faster than with eEF2 alone, suggesting that binding of eEF3 to the ribosome stabilizes a ribosome conformation that is somewhat more active in peptidyl transfer reaction with Pmn, possibly by stabilizing peptidyl-tRNA in the exit tunnel (see cryo-EM data below). Also, consistent with the results of the tripeptide synthesis (Figure 1D), eEF3 alone appears to catalyze translocation, but not to the same extent as in the presence of eEF2 (Figure 2B), which is not increased further with the pre-incubation time (not shown).

\section{E-site tRNA release by eEF3 does not require ternary complex binding}


The final step (3) of translocation entails dissociation of deacylated tRNA from the E site (Figure 2A). To monitor this step in real time, we used a rapid kinetics assay that follows a fluorescence change of tRNA ${ }^{\mathrm{fMet}}$ labeled by fluorescein, $\mathrm{tRNA}^{\mathrm{fMet}}(\mathrm{Flu})$, upon dissociation from the ribosome (Belardinelli et al., 2016). The tRNA is functionally active in the yeast translation system to the same extent as a non-modified $\mathrm{tRNA}_{\mathrm{i}}{ }^{\mathrm{Met}}$, as validated by the tripeptide formation assay (Figure S2G). When we rapidly mixed $80 \mathrm{~S} 2 \mathrm{C}$ containing tRNA ${ }^{\mathrm{fMet}}(\mathrm{Flu})$ and Met-Phe-tRNA ${ }^{\text {Phe }}$ with eEF2, eEF3, and TC-Val in the stopped-flow apparatus, fluorescence decreases (Figure 2C, green trace). At the starting point of the experiment, $\mathrm{tRNA}^{\mathrm{fMet}}(\mathrm{Flu})$ resides in the $\mathrm{P} / \mathrm{P}$ or $\mathrm{P} / \mathrm{E}$ state, while the fluorescence change reports how much $\mathrm{tRNA}^{\mathrm{fMet}}(\mathrm{Flu})$ has been released from the ribosome. In the process of reaction, addition of eEF2 and eEF3 together with TC-Val leads to a first translocation, Val-tRNA ${ }^{\text {Val }}$ binding, next peptide bond formation, followed by a second translocation and the dissociation of tRNA ${ }^{\text {Met }}$ from the $\mathrm{E}$ site. Thus, the fluorescence change over the time course with eEF2, eEF3, and TC-Val gives the maximum signal difference anticipated upon E-site tRNA dissociation (Figure 2C, green trace). When a similar experiment is carried out in the absence of TC-Val, only the first translocation can occur; we observe that in this case, the fluorescence of tRNA ${ }^{\text {Met }}$ decreases considerably, but not to the same extent as when TC-Val is included (Figure $\mathbf{2 C}$, purple trace). Addition of eEF3 alone or eEF2 alone resulted in only a very small fluorescence change (Figure 2C, blue and red traces). These results suggest that eEF2 and eEF3 together facilitate the movement of the E-site tRNA and its ultimate dissociation from the E site. By contrast, eEF2 alone can catalyze partial tRNA displacement (that promotes Pmn reactivity, Figure 2B), but is incapable of promoting dissociation of the E-site tRNA during the short (physiologically relevant) time window.

We further tested whether the tRNA dissociation from the E site and TC binding to the A site are directly coupled. We carried out experiments where we incubated $80 \mathrm{~S} 2 \mathrm{C}$ (with tRNA ${ }^{\text {fMet }}(\mathrm{Flu})$ ) with eEF2 and eEF3 or with eEF2 alone for an extended period (15 min) before adding TC-Val. The experiment with eEF2 and eEF3 gives the magnitude of the signal change due to the second translocation and dissociation of tRNA ${ }^{\text {fMet }}(\mathrm{Flu})$ from the E site (Figure 2D, green trace). No fluorescence change is observed with eEF2 alone (Figure 2D, red trace), although from the kinetics of tripeptide formation we observe that 
under these conditions, eEF2 alone promotes partial tRNA translocation (Figure 1D). These experiments clearly establish that binding of the cognate TC into the A site is not coupled to the dissociation of the E-site tRNA, in contrast to previous suggestions (TrianaAlonso et al., 1995). Thus, the role of eEF3 is to facilitate the late stages of translocation by promoting the dissociation of the deacylated tRNA from the E site, but has little effect on A-site binding (Figure 1D and 2D).

\section{Ribosome profiling of eEF3-depleted yeast cells}

Having shown in vitro that eEF3 is essential for late steps of translocation, we corroborated our findings in vivo using ribosome profiling. Because eEF3 is an essential gene in budding yeast, to observe its function in vivo we developed a system to conditionally deplete eEF3 using transcriptional shutoff and an auxin degron tag (mAID) as described previously (Nishimura et al., 2009; Schuller et al., 2017). In the eEF3 depletion strain (dubbed eEF3d), expression of eEF3 is controlled by a GAL1 promoter that is turned off in the presence of glucose. Following a switch to glucose in the media and the addition of auxin, eEF3 was barely detectable by immunoblotting after 6 hours (Figure S3A). As anticipated based on its essential nature, depletion of eEF3 reduces cell growth compared to wild type (WT) cells (Figure S3B).

We prepared libraries of ribosome footprints from WT and eEF3d strains after $8 \mathrm{~h}$ of conditional growth. Our recent study showed that using combinations of elongation inhibitors for library preparations allows us to distinguish three distinct ribosome functional states - pre-accommodation (PreAcc), pre-peptide bond formation (PrePT), and pre-translocation (PreTrans) — from different sized ribosome-protected footprints (RPFs) (Wu et al., 2019) (Figure 3A). In a first set of experiments, we prepared libraries with a combination of cycloheximide (CHX) and tigecycline (TIG); CHX blocks translocation whereas TIG blocks aa-tRNA accommodation in the A site as indicated (Jenner et al., 2013; Schneider-Poetsch et al., 2010). In these libraries, 21 nt RPFs correspond solely to ribosomes with an empty A site on the small subunit, e.g. in a PreAcc state, whereas $28 \mathrm{nt}$ RPFs predominantly correspond to ribosomes trapped in a PreTrans state (Figure 3A). In WT cells, we observe a distribution of 21 and 28 nt RPFs, genomewide, suggesting that no single step of translation elongation is rate-limiting under normal 
circumstances, but that instead ribosomes are found in a relatively even distribution of functional states (Figure 3B, black line). By contrast, in eEF3d cells, we observed a drastic reduction in $21 \mathrm{nt}$ RPFs (and a concomitant increase in $28 \mathrm{nt}$ RPFs) when compared to WT cells (Figures 3B and S3C), indicating the accumulation of PreTrans state ribosomes. First, this observation establishes a role for eEF3 in translation elongation, as its depletion causes a genome-wide change in the distribution of ribosome functional states. More specifically, the reduction in $21 \mathrm{nt}$ RPFs in eEF3d cells is indicative of an increase in ribosomes with A sites occupied by aa-tRNAs. These data suggest that either peptide-bond formation or translocation becomes rate limiting in vivo in the absence of eEF3.

We next prepared libraries using a combination of CHX and anisomycin (ANS); ANS blocks peptidyl transfer (Grollman, 1967). $28 \mathrm{nt}$ RPFs report solely on ribosomes in a PreTrans state, whereas $21 \mathrm{nt}$ RPFs correspond to ribosomes either in a PreAcc or PrePT state due to dissociation of the unreacted aa-tRNA from the A site (Figure 3C). As anticipated, libraries prepared from WT cells revealed a distribution of 21 and $28 \mathrm{nt} \mathrm{RPFs,}$ but with a greater number of $21 \mathrm{nt}$ RPFs than for libraries prepared with $\mathrm{CHX}+\mathrm{TIG}(\mathrm{Wu}$ et al., 2019). We further found that the footprints from eEF3d cells are almost devoid of 21 nt RPFs when compared with the WT samples prepared with CHX+ANS (Figure 3D). In addition, the association between eEF1A (or eEF2) and the ribosome is not influenced upon eEF3 depletion as monitored by polysome profiles and immunoblotting (Figure S3D); these data indicate that neither tRNA selection nor peptide bond formation is rate limiting in the absence of eEF3. Taken together, our experiments argue that eEF3 promotes the translocation step of elongation, consistent with our in vitro data (Figure 2B).

\section{Codon level analysis of ribosome functional states in the eEF3d strain}

In addition to global changes in the distribution of ribosome functional states, we asked whether ribosomes are disproportionally stalled at specific sites. By calculating A-site ribosome occupancies (i.e. pause scores) of $21 \mathrm{nt}$ RPFs (from samples prepared with $\mathrm{CHX}+\mathrm{TIG}$ ) at all 61 sense codons, we observed a distribution of pause scores in WT cells, and a drastic reduction in this distribution in eEF3d cells; as a result, we see a nearly horizontal line comparing these different distributions with a slope of 0.18 (Figure 3E). Different ribosome occupancies on codons, i.e. "codon optimality", is thought to be 
determined by several factors including codon usage, cellular tRNA concentrations, and the efficiency of wobble pairing (dos Reis et al., 2004; Percudani et al., 1997). For example, pause scores at rare codons (such as CGA) are substantially higher than those at common codons (such as AGG) in WT cell (Figures 3E and 3F), as shown in earlier studies (Weinberg et al., 2016; Wu et al., 2019); these differences are however substantially smaller in eEF3d cells (Figure 3F, quantified by variance). Importantly, because the calculation of ribosome occupancies (pause scores) at codons from ribosome profiling experiments relies on internal normalization (i.e. an increase in ribosome occupancy at one codon necessarily reduces those at other codon(s)), we do not interpret the absolute values of these numbers in any manner. Given that $21 \mathrm{nt}$ RPFs from samples prepared with $\mathrm{CHX}+\mathrm{TIG}$ represent ribosomes poised for decoding (Figure 3A), these observations suggest that in the absence of eEF3, a step preceding decoding has become rate limiting, thereby reducing the decoding rate in the A site in a global manner such that codon specific differences are lost (Figure 3F, e.g. AGG vs. CGA).

We similarly characterized $28 \mathrm{nt}$ RPFs in libraries prepared with $\mathrm{CHX}+\mathrm{ANS}$ (Figure 3D), focusing on whether there are any sequence motifs that tend to be enriched in the PreTrans state (Figure 3C); in these libraries, 28 nt RPFs represent ribosomes trapped in a PreTrans state. First, in the WT cells, we found a distribution of codon-specific occupancies that differ by as much as 3-fold; among the most enriched codons are those that correspond to those encoding proline (P, purple) and glycine (G, green) (Figure 3G). Second, as for our analysis of the 21 nt RPFs (Figure 3E), we observed a loss of codon specific differences in ribosome occupancy when eEF3 is depleted, as evidenced by a flattening of the correlation plot (slope 0.25 ). Further analysis of peptide motifs revealed that among 8000 tripeptides, the most enriched sequences were those encoding collections of $\mathrm{P}, \mathrm{G}$ and $\mathrm{D}$ amino acids (Figures $\mathbf{3 H}$ and S3E). These observations are reminiscent of sequences that depend on eIF5A for peptide bond formation (Schuller et al., 2017), though here we are seeing that these same sequences are slowing the translocation step of protein synthesis. Consistent with the results of in vitro biochemistry, we conclude that eEF3 contributes to the translocation step of protein synthesis, and in its absence, an early step in the process becomes rate limiting. 


\section{An ex vivo structure of eEF3 on the yeast $80 \mathrm{~S}$ ribosome}

To investigate a physiologically-relevant structure of eEF3 on the yeast $80 \mathrm{~S}$ ribosome at different phases of the elongation cycle, we opted to obtain eEF3-80S complexes by employing an ex vivo pull-down approach. To do this, a S. cerevisae BY4741 strain expressing C-terminally TAP-tagged eEF3 was grown to log phase and eEF3-80S complexes were isolated using IgG-coupled magnetic beads (Figure 4A). The complex was eluted by TEV protease cleavage of the TAP-tag in the presence of non-hydrolyzable ADPNP and the resulting eluate was crosslinked with glutaraldehyde and directly applied to cryo-grids. It is important to note that in the absence of crosslinker, reconstructions yielded 80S ribosomes, but without additional density for eEF3, suggesting that eEF3 dissociated from the ribosomes during grid application. The ex vivo eEF3-80S complex was then analyzed using single particle cryo-EM. After 2D classification, 211,727 ribosomal particles were initially sorted into eight classes, two of which had strong density for eEF3 (I: class 1 and 2), one had density for eEF2 and a weak density for eEF3 (II: class 3), two had weak density for eEF3 (III: class 4 and 5) and the remaining three classes (IV: class 6-8) were poorly defined and had low resolution (Figure S4). The two classes (I: class 1 and 2) with strong density for eEF3 were combined and a local mask was applied around eEF3 and a second round of focused sorting and refinement was undertaken, eventually yielding a cryo-EM reconstruction of the eEF3-80S complex (Figure 4B and Video S1) with an average resolution of $3.3 \AA$ (Figure S5A). Local resolution calculations reveal that while the majority of the ribosome is well-resolved (Figure S5B), the resolution of eEF3 varies, with ribosome-interacting regions being better resolved (3-4 $\AA$ ) than the peripheral regions (4-6 $\AA$ ) (Figure S5D). As observed in the previous eEF3-80S structure (Andersen et al., 2006), density for eEF3 spans between the head of the 40S subunit and the central protuberance of the 60S subunit (Figure 4B). We therefore also employed multi-body refinement (Figure S4), which led to slight improvements in the local resolution for the region of eEF3 that interacts with the head of the 40S subunit (Figure S5E-H). The resolution allowed an accurate domain-by-domain fit of the previous $2.4 \AA$ X-ray structure of yeast eEF3 (Andersen et al., 2006) into the cryo-EM density map of the eEF3-80S complex (Figure S6A-C and Video S1). Electron density for many sidechains could be observed, especially bulky and aromatic amino acids, enabling us to generate an 
initial model for the N-terminal HEAT domain, as well as C-terminal ABC1/2 and CD. By contrast, the more peripheral $4 \mathrm{HB}$ domain was poorly resolved and was modeled only at the secondary structure level, and the final 68 residues of the C-terminus had no density and were therefore not included in the model. We note that electron density consistent with ATP (or ADPNP) was observed in the active sites of the $\mathrm{ABC} 1$ and $\mathrm{ABC} 2$ nucleotide binding domains (NBDs) (Figure S6D-E). This is consistent with the closed conformation we observed for $\mathrm{ABC} 1$ and $\mathrm{ABC} 2 \mathrm{NBDs}$, which is similar to the closed conformations adopted in the 70S-bound ABCF protein VmlR from B. subtilis (Crowe-McAuliffe et al., 2018) and archaeal 40S-ABCE1 complex (Heuer et al., 2017), but distinct from the open conformation observed for the free state of ABCE1 (Karcher et al., 2008) (Figure S7A-D). Indeed, modelling of an open conformation of eEF3 on the ribosome suggests that it is incompatible with stable binding, leading to either a clash of the ABC2-CD with the central protuberance of the $60 \mathrm{~S}$ subunit or dissociation of the ABC1-HEAT-4HB domain from the 40S subunit (Figure S7E-G). These observations support the idea that eEF3 binds to the ribosome in the "closed" ATP-bound conformation and that ribosome-stimulated hydrolysis of ATP to ADP would promote eEF3 dissociation.

\section{Interaction of eEF3 on the yeast $80 \mathrm{~S}$ ribosome}

The overall geometry of eEF3 on the $80 \mathrm{~S}$ ribosome is similar to that reported previously for eEF3 (Andersen et al., 2006), as well as its homologue in yeast New1p (Kasari et al., 2019b), namely, oriented such that the N-terminal HEAT and 4HB interact with the 40S subunit, whereas the C-terminal CD contacts exclusively the 60S subunit (Figure 4C-E). As with New1p (Kasari et al., 2019b), the majority of the contacts with the 40S subunit are established by the HEAT domain of eEF3 with components of 40S head including the tip of ES39S of the 18S rRNA as well as r-proteins uS13 and uS19 (Figure 4D-E and S8AD). The majority of the interactions involve positively charged residues located within the loops linking the HEAT repeat $\alpha$-helices, such as Lys101 (loop $\alpha 3-\alpha 4)$, Lys141 (loop $\alpha 5-$ a6) and Lys182 (loop $\alpha 7-\alpha 8$ ) that are in close proximity to the nucleotides A1360 and U1361 of ES39S (Figure S8C). Interactions are also observed between the HEAT domain loops (Gln143, Asp180, Glu184) with charged residues (Gln25, Arg130, Asp127 and Arg134) of eS19 (Figure S8C). The distal end of the HEAT domain (conserved residues 
302-307, Glu304, Arg306, Glu307) also establishes interactions with uS13 in the vicinity of His78 and Arg80 (Figure S8D). The 4HB and ABC1 domains of eEF3 do not interact with any ribosomal components, whereas $\mathrm{ABC} 2$ bridges the intersubunit space by forming protein-protein interactions with uS13 on the 40S involving Arg871 and Arg872 (Figure S8D) and uL5 and uL18 on the 60S subunit (Figure S8E-F). In addition, the loop between the $\beta 2$ and $\beta 3$ strands of the CD contains positively charged residues (Arg802, Arg803, Lys804, Lys806 as well as Asn807) that appear to interact with nucleotides U32-G41 of the 5S rRNA (Figure S8F).

\section{eEF3 facilitates E-site tRNA release by opening the L1 stalk}

To address which functional states of the ribosome are stably bound by eEF3, we implemented additional rounds of 3D classification of the classes with strong density for eEF3 (group I: classes 1 and 2), but with a focus on the L1-stalk and tRNAs (Figure S4). This yielded four subclasses (Ia-Id) that all exhibited strong density for eEF3, but differed with respect to their functional state. Of these four classes, two pre-translocation (PRE) states were observed that could be refined to $3.5 \AA$ and $4.0 \AA$, respectively (Figure $\mathbf{5 A}$, 5B). PRE-1 contained classical A- and P-site tRNAs, whereas PRE-2 contained a classical A-site tRNA and a hybrid P/E-site tRNA. In both PRE-1 and PRE-2, density for the nascent polypeptide chain could be observed extending from the A-site tRNA and entering into the ribosomal exit tunnel (Figure 5A, 5B). Since eEF3 has been suggested to play a role in the dissociation of E-site tRNA from the ribosome, the observation of eEF3 bound to various PRE-state ribosomes was surprising and the implication of this with respect to dissociation of eEF3 from the ribosome will be discussed later where we focus on the identification of PRE-3, PRE-4 and POST-1 states that lack clear density for eEF3. In addition to the PREstate complexes, two post-translocation (POST) state ribosomes were obtained, POST-2 and POST-3, which could be refined to $3.9 \AA$ and $3.8 \AA$, respectively (Figure 5C, 5D, S5). POST-2 contained classical P- and E-site tRNAs, whereas POST-3 contained a P-site tRNA, but lacked an E-site tRNA (Figure 5C, 5D). In both the POST-2 and POST-3 states, density for the nascent polypeptide chain could also be visualized attached to the P-site tRNA (Figure 5C, 5D). The presence and absence of E-site tRNA in the POST-2 and POST-3, respectively, suggested that these complexes could represent the states before and 
after eEF3-mediated E-site tRNA release, respectively. Consistent with this hypothesis, the L1 stalk is observed with an "in" conformation in POST-2 that would prohibit release of the E-site tRNA from the ribosome, whereas in POST-3 the L1 stalk has moved into an "out" conformation that would enable E-site tRNA dissociation (Figure 5E, 5F). In POST2, we observe no direct interaction between eEF3 and the E-site tRNA, but rather between the CD of eEF3 and the L1 stalk (Figure 5G, 5H). Specifically, the contact between eEF3 and the L1 stalk is from the tip of the CD with domain II of the $\mathrm{uL1}$ protein (Figure 5H). Unfortunately, the L1 region is poorly ordered due to high flexibility, nevertheless, it appears that the interactions are dominated by charge complementarity between the conserved glutamates (Glu790, Glu819 and Glu826) in the CD that are in close proximity to a highly conserved lysine-rich region (Lys91-92, Lys95, Lys97-98, Lys101-102, Lys105-106) present in domain II of uL1 (Figure 5H). The large number of lysine residues in this region of $\mathrm{uL} 1$ is intriguing since $\mathrm{CD}$ are best known from chromatin remodelling proteins where they bind to methylated lysines (me-K) present in histone tails (Nielsen et al., 2002; Taylor et al., 2007; Yap and Zhou, 2011). Methylation of few non-histone proteins has been reported, one of which is the $\mathrm{uL} 1$ protein that is methylated on a single lysine (me-K46) (Webb et al., 2011); however, K46 is located in domain I of uL1, far from the CD interaction site (Figure S9). Moreover, the hydrophobic pocket that is generally formed by three aromatic residues to interact with the me-K, does not appear to be present in eEF3 (Figure S9).

Collectively, our findings imply that eEF3 is likely to facilitate E-site tRNA release (as we observe biochemically, Figure 2D) indirectly by promoting the transition of the L1 stalk from the "in" to the "out" conformation (Figure 5E, 5F), rather than from directly using the $\mathrm{CD}$ to dislodge the E-site tRNA from its binding site.

\section{eEF3 binds stably to non-rotated ribosomal states}

We noted that although eEF3 bound stably to a mixture of different PRE and POST states (Figure 5A-D), these ribosomal states nevertheless had a common feature in that they are all non-rotated i.e. they have a non-rotated $40 \mathrm{~S}$ with respect to the $60 \mathrm{~S}$ subunit. This nonrotated state is critical for eEF3 to establish simultaneous interactions with the ES39S, uS13 and eS19 on the head of the 40S as well as the 5S rRNA, uL5 and uL18 on the 60S 
subunit, as described for the high resolution eEF3-80S complex (Figure 4D, 4E and S8), thereby providing an explanation for the strong density observed for eEF3 in the nonrotated PRE-1/-2 and POST-2/-3 state. To explore this idea further, we also analyzed the classes where the density for eEF3 was poorly resolved (Figure S5, group II and III). Refinement of group II (class 3) to an average resolution of $4.1 \AA$ yielded an eEF2-bound ribosome with P- and E-site tRNAs (POST-1) and poorly defined density for eEF3 (Figure 6A). In addition to intersubunit rotation by $8.5^{\circ}$, we also observed a counter-clockwise (towards the E-site) head swivel of $20^{\circ}$ (Figure 6B). As expected, the rotation and headswivel movements have a large impact on the eEF3 binding site, namely, moving the 40S interaction partners, such as ES39S, eS19 and uS13, by $16 \AA$ for the rRNA, $17 \AA$ for eS19 up to $27 \AA$ for uS13 relative to the $60 \mathrm{~S}$ and their position in the non-rotated ribosome (e.g. POST-3) (Figure S10A-B). Closer inspection of the density for eEF3 in POST-1 indeed revealed that the majority of the density remains associated with the $60 \mathrm{~S}$ and that both rotation and swiveling causes a loss of connectivity between eEF3 and the head of the 40S (Figure S10C). Since there is defined density for eEF3 in the non-rotated POST-2 and POST-3, but eEF3 is disordered in the eEF2-bound rotated POST-1 state, this indicates that stable binding of eEF3 occurs during or subsequent to eEF2 dissociation from the ribosome when the $40 \mathrm{~S}$ resets and the ribosome returns to a non-rotated and non-swivelled conformation. We suggest that eEF3 facilitates late stages of translocation by stabilizing the non-rotated state after eEF2 has promoted the movement of the tRNA-mRNA complex (Figure 1D).

\section{Intersubunit rotation leads to unstable eEF3 binding on the ribosome}

Further sorting and refinement of group III (Figure S4, classes 4 and 5) yielded two distinct PRE states, namely, PRE-3 containing a classical A-site tRNA and a hybrid P/E-site tRNA, and PRE-4 containing hybrid A/P- and P/E-site tRNAs (Figure 6C, 6D), which could be refined to average resolutions of $4.2 \AA$ and $3.3 \AA$, respectively. Analogous to PRE-1 and PRE-2, both PRE-3 and PRE-4 states also had density for the nascent polypeptide chain, but extending from the A-site tRNA (rather than the P-site tRNA) into the ribosomal exit tunnel (Figure 6C, 6D). However, unlike PRE-1 and PRE-2, the 40S in PRE-3 and PRE4 was rotated by $9.6^{\circ}$ and $11^{\circ}$ relative to the $60 \mathrm{~S}$ subunit (Figure 6E, 6F). In the PRE-3 
state, little to no density for eEF3 was observed (Figure 6C), suggesting that the factor had dissociated from the ribosome during sample and/or grid preparation. While significant density was observed for eEF3 in PRE-4, the density was poorly resolved (Figure 6D). Analogous to POST-1, the rotation observed in PRE-3 and PRE-4 appears to also disrupt the interactions with the 40S subunit such that the majority of the eEF3 density is connected to the 60S subunit (Figure S10D-I). Collectively, this supports the suggestion that stable binding of eEF3 to the ribosome requires a non-rotated state and that intersubunit rotation leads to destabilization of eEF3 binding (Figure 6C and S10F). eEF3 remains stably bound to the ribosome subsequent to E-site tRNA release, and is still stably bound when the next aminoacyl-tRNA has accommodated into the A-site and undergone peptide bond formation. Rather it appears that the critical step that leads to conformational rearrangements in eEF3 is the transition from non-rotated to rotated states. In this respect, it is interesting to note that PRE-3 has the same tRNA arrangement as PRE-2, namely, both containing a classical A-site tRNA with nascent chain and a deacylated hybrid P/E-site tRNA, yet adopt completely different rotational states (Figure 5B, 6C). This is somewhat surprising since formation of hybrid states in higher eukaryotes is generally assumed to be concomitant with intersubunit rotation (Behrmann et al., 2015; Budkevich et al., 2011; Svidritskiy et al., 2014), however, we note that subunit rotation and tRNA movement is only loosely coupled on bacterial ribosomes (Fischer et al., 2010). Since rotation of PRE2 would result in a state similar to PRE-3, we suggest that subunit rotation is what triggers the low affinity form of eEF3, effectively leading to unstable ribosome binding. 


\section{Discussion}

Collectively, our findings suggest a model for the role of eEF3 during the translocation elongation cycle (Figure 7A-G and Video S2). The ability to capture four different PRE and three different POST states of the ribosome by co-immunoprecipitation of tagged-eEF3 suggests that $\mathrm{eEF} 3$ is omnipresent during translation elongation in yeast, which is consistent with the similar copy number between eEF3 and ribosomes (Firczuk et al., 2013). Our biochemical data show that the main function of eEF3 is to accelerate the Esite tRNA release from the ribosome during late stages of mRNA-tRNA translocation (Figures 1 and 2). These in vitro findings are in strong agreement with the in vivo ribosome profiling experiments. Similar to previous studies where eEF3 was depleted in the cell (Kasari et al., 2019a), we observe general defects in translation elongation. However, our application of high-resolution ribosome profiling, using combinations of elongation inhibitors for library preparation, allowed us to follow ribosomes trapped in distinct functional states and thereby identify the specific elongation defect (Figure 3). In particular, we find that eEF3 depletion enriched ribosomes trapped in a pre-translocation state (28 nt RPFs), fully consistent with the observation that eEF3 promotes a late step in translocation that depends on E-site tRNA release. A strong prediction of the biochemical and ribosome profiling results is that the ribosomes that accumulate in cells on eEF3 depletion would contain three separate tRNAs.

Cryo-EM structures suggest two mechanisms by which eEF3 can facilitate translocation: by favoring the transition towards the non-rotated conformation of the ribosome and operating the conformation of the L1 stalk. As in all kingdoms of life, yeast eEF2 stabilizes the rotated state of the ribosome and accelerates the movement of the peptidyl-tRNA from the A to the P site (Figure 7D-E). In bacteria, the eEF2 homolog EFG promotes the small subunit head swiveling, which allows the mRNA-tRNAs to move relative to the large subunit (Belardinelli et al., 2016; Wasserman et al., 2016; Zhou et al., 2014). Resetting the swiveled conformation triggers the release of EF-G and the E-site tRNA, which occurs rapidly on a millisecond time scale (Belardinelli et al., 2016), and does not require auxiliary factors. The present structures suggest which features of the mechanism are conserved in yeast. Similar to EF-G, eEF2 catalyzes head swiveling and mRNA-tRNA translocation (Figure 7E), whereas dissociation of the factor and back- 
swivel occur in a later step (Figure 7A-B). The cryo-EM observation of discrete POST-1 and POST-2 states suggests that this transition is relatively slow in yeast cells, even in the presence of eEF3. Stabilization by eEF3 of the non-rotating state may accelerate the reaction and contribute to the directionality of translocation. Since we do not observe any difference in the association of eEF2 with ribosomes upon eEF3 depletion (Figure S3D), we do not think that eEF3 plays an important role for eEF2 dissociation. Similarly, small differences in the rates of peptide bond formation (Figure 1D and 2B) can be attributed to the effect of eEF3 on the ribosome dynamics, but do not have a major effect in vivo (Figure 3C-F). By contrast, we observe dramatic differences in the rates of E-site tRNA release in the presence and absence of eEF3 (Figure 2D).

In contrast to bacterial ribosomes, which release the E-site tRNA quickly (Belardinelli et al., 2016; Uemura et al., 2010), dissociation of deacylated tRNA from yeast ribosome in the absence of eEF3 is very slow, occurring in the seconds range (this paper and (Garreau de Loubresse et al., 2014)). Even in the presence of eEF3, E-site tRNA release is observed as a separate step from POST-2 to POST-3 state (Figure 7F-G). In our POST2 state, the CD of eEF3 is seen to directly contact the L1 stalk, but not the E-site tRNA, suggesting that dissociation of the E-site tRNA by eEF3 is facilitated by shifting the L1 stalk from an "in" to an "out" conformation (Figure 7F-G), as was proposed previously (Andersen et al., 2006; Triana-Alonso et al., 1995). We note, however, that we do not observe release of the E-site tRNA to be dependent on binding of the ternary complex to the A-site (Figure 2C), as suggested previously (Triana-Alonso et al., 1995). We also do not observe the presence of eEF1A in any of our cryo-EM states, and depletion of eEF3 does not lead to accumulation of eEF1A (or eEF2) on the ribosome (Figure S3D). Rather, our in vivo eEF3-depletion studies suggest that the ribosomes become blocked with all three tRNA binding sites being occupied (Figure 7H). As biochemical experiments show that peptidyl transfer can occur in the absence of eEF3 (Figure 1D), we envisage that ribosomes are trapped in the POST-2 state with deacylated tRNAs in the E- and P-sites and a peptidyl-tRNA in the A site. Importantly, the subsequent translocation step would not be possible, probably because the presence of deacylated tRNA at the E-site inhibits the ribosome rotation and tRNA movement. Such a state with three tRNAs on the ribosome would be consistent with the loss of $21 \mathrm{nt}$ RPFs in the ribosome profiling experiments 
observed when eEF3 is depleted from the cells (Figures 3B and 3D). While it has been shown by smFRET that bacterial ribosomes with three tRNAs assume a partially rotated state due to slow kinetics of E-site tRNA release which hinders translocation (Choi and Puglisi, 2017), it is tempting to speculate that fungi may have evolved to utilize eEF3 to overcome this kinetic hurdle, promoting rapid translation.

eEF3 has a marked binding preference for the non-rotated states of the ribosome, e.g. PRE-1 and PRE-2, as well as POST-2 and POST-3 states, and becomes destabilized in the rotated PRE-3 and PRE-4 that arise when the A- and/or P-site tRNAs move into hybrid sites (Figure 7C, 7D). Similarly, eEF3 does not stably interact with the eEF2-bound POST1 state bearing translocated P-and E-site tRNAs (Figure 7E, S10C). Comparison of rotated and non-rotated ribosome conformations reveals that subunit rotation disturbs the interactions between eEF3 and the $40 \mathrm{~S}$ subunit, providing a rationale for the destabilization (Figure S10). By contrast, the back-rotation and back-swivel of the head that accompanies eEF2 dissociation yields a classical non-rotated POST-2 state with P- and E-site tRNAs that is an optimal substrate for stable binding of eEF3 (Figure 7F). In this state, we observe that eEF3 adopts a closed conformation with two molecules of ATP bound within the $\mathrm{ABC} 1$ and $\mathrm{ABC} 2$ NBDs (Figure S6D-E). Stable binding of eEF3 to the ribosome during decoding, e.g. at the transition from POST-3 to PRE-1 state may affect the relative rates of decoding reactions, which may explain the effect of eEF3 on translation fidelity (Uritani and Miyazaki, 1988) and account for the observed genetic interactions between eEF1A and eEF3 (Anand et al., 2006; Anand et al., 2003)

In addition to providing insight into the function of eEF3, our study fills the gap for various missing structures of the yeast translation elongation cycle (Figure 7 and Video S2). While yeast PRE-1 (Figure 7A) and PRE-4 (Figure 7D) states have been reported recently in yeast (Buschauer et al., 2020; Ikeuchi et al., 2019; Matsuo et al., 2020), those structures did not contain eEF3. We also observe the novel non-rotated PRE-2 (Figure 7B) as well as canonical rotated PRE-3 states (Figure 7C). PRE-1, PRE-3 and PRE-4 are similar to classical and rotated states observed in mammalian systems (Behrmann et al., 2015; Budkevich et al., 2011). While there have been multiple structures of yeast eEF2 on the ribosome (Gomez-Lorenzo et al., 2000; Pellegrino et al., 2018; Sengupta et al., 2008; Spahn et al., 2004; Taylor et al., 2007), none contained the physiological configuration of 
two tRNAs being translocated by eEF2, as observed in our POST-1 state (Figure 7E). We note however, that similar functional states to POST-1 have been observed in rabbit and human systems (Behrmann et al., 2015; Flis et al., 2018). Finally, although an in vitro reconstituted POST-3 state with eEF3 (Figure 7G) has been reported at $9.9 \AA$ resolution (Andersen et al., 2006), here we present an ex vivo POST-3 at $3.8 \AA$ as well as additionally capturing POST-2 state with eEF3 and E-site tRNA (Figure 7F).

Collectively, our biochemical, genetic and structural analysis sheds light into the critical function of eEF3 during translation in yeast. Ultimately, rapid translation in yeast requires fast movement of the tRNAs through the ribosome and their release from the $\mathrm{E}$ site. Here we show that eEF3 fulfills both roles by facilitating late steps in tRNA translocation and by inducing the L1 stalk to adopt an out conformation allowing E-site tRNA release. One question for the future is to understand why a factor such as eEF3 that is essential for translation yeast does not appear to have a homolog in higher eukaryotes, such as humans. 


\section{FIGURES}

A
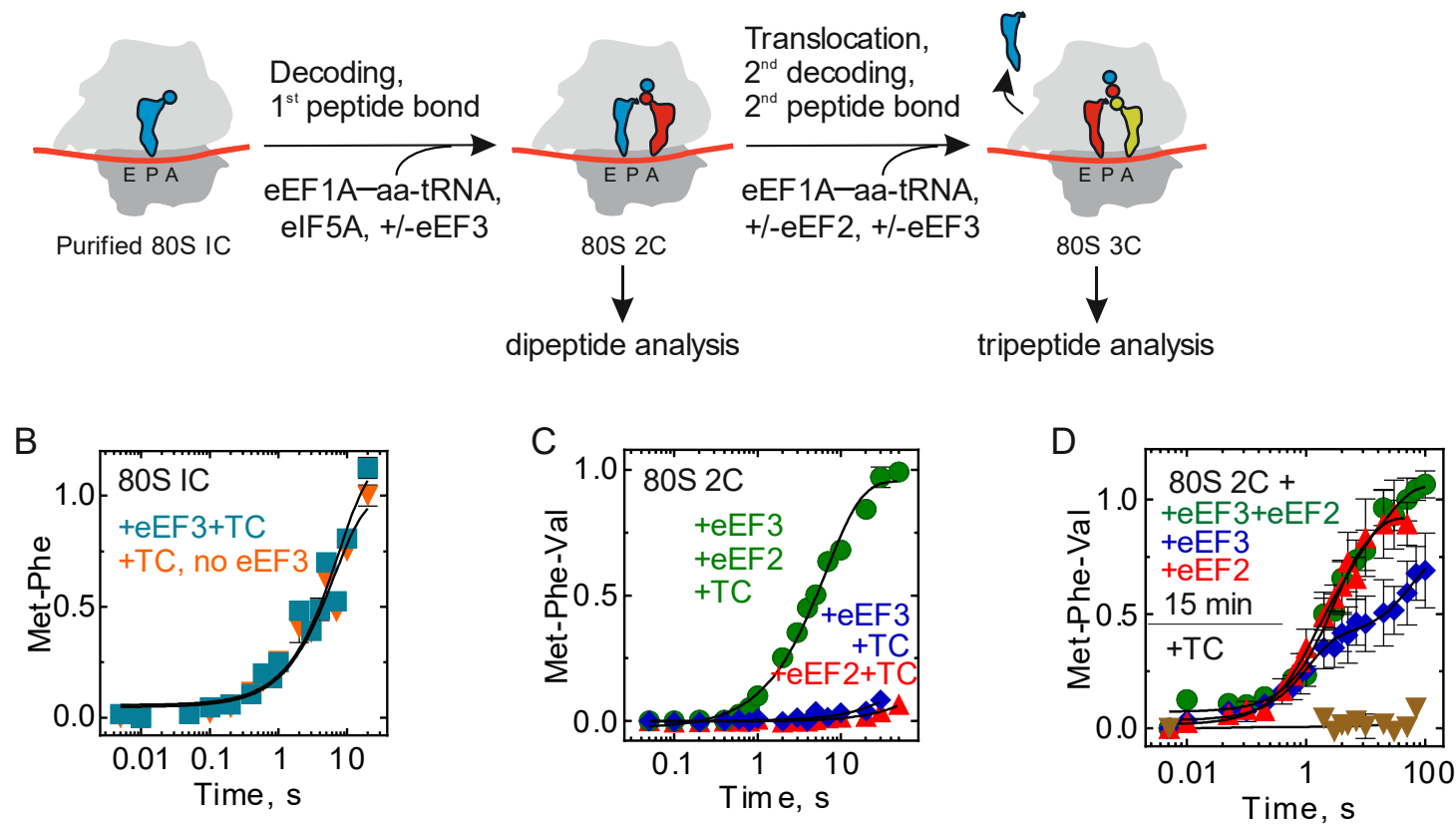

Figure 1. eEF3 is essential for tri- \& polypeptide formation (A) Schematic of the translation elongation cycle as studied here. (B) Met-Phe formation monitored upon rapidly mixing initiation complexes (80S IC) with ternary complexes eEF1A-GTP- $\left[{ }^{14} \mathrm{C}\right] \mathrm{Phe}-$ tRNA $^{\text {Phe }}$ in the presence (cyan, $0.15 \pm 0.02 \mathrm{~s}^{-1}$ ) or absence (orange, $0.13 \pm 0.02 \mathrm{~s}^{-1}$ ) of eEF3 in a quench-flow apparatus, and the extent of peptide formation was analyzed by HPLC and radioactivity counting. Data presented as mean $\pm S E M$ of $n=3$ biological replicates. (C) Met-Phe-Val formation monitored upon rapidly mixing $80 \mathrm{~S}$ complexes carrying MetPhe-tRNA $^{\text {Phe }}(80 \mathrm{~S} 2 \mathrm{C})$ with ternary complexes eEF1A-GTP- $\left[{ }^{14} \mathrm{C}\right]$ Val-tRNA ${ }^{\mathrm{Val}}$ in the presence of eEF2 and eEF3 (green, $0.15 \pm 0.01 \mathrm{~s}^{-1}$ ), eEF2 (red) or eEF3 (blue). Data presented as mean \pm SEM of $n=3$ biological replicates. (D) Met-Phe-Val formation monitored with $80 \mathrm{~S} 2 \mathrm{C}$ incubated with eEF2 and eEF3 (green, $0.4 \pm 0.14 \mathrm{~s}^{-1}$ ), eEF2 (red, $1.0 \pm 0.5 \mathrm{~s}^{-1}$ ) or eEF3 (blue) for $15 \mathrm{~min}$ before adding with ternary complexes eEF1A-GTP$\left[{ }^{14} \mathrm{C}\right]$ Val-tRNA ${ }^{\mathrm{Val}}$ and monitoring the kinetics of Val incorporation. Data presented as mean $\pm \mathrm{SEM}$ of $\mathrm{n}=3$. 


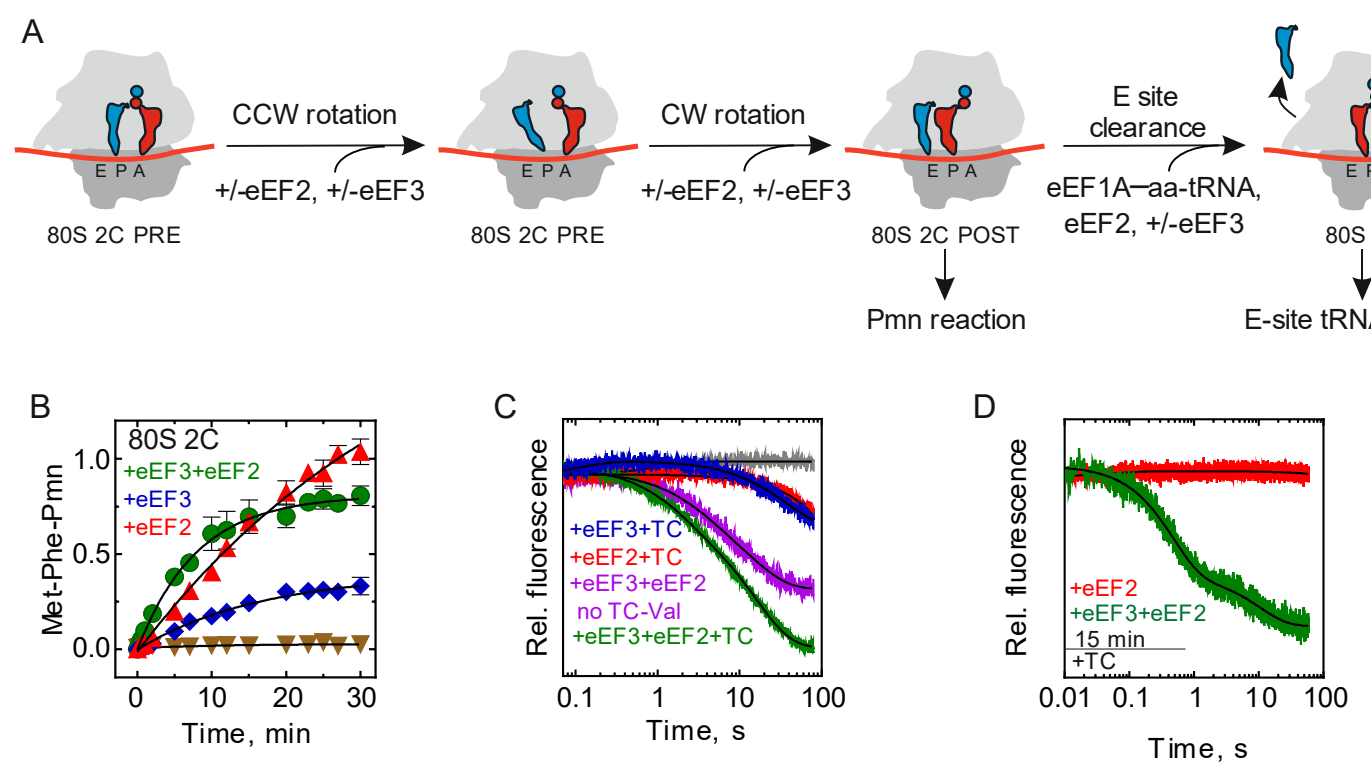

Figure 2. eEF3 in tRNA movement during translocation and E-site clearance

(A) Schematic of tRNA translocation. (B) mRNA-tRNA translocation monitored in a timeresolved Pmn assay upon mixing 80S 2C with Pmn in the absence (brown triangles) or presence of eEF2 and eEF3 (green, $0.13 \pm 0.01 \mathrm{~min}^{-1}$ ), eEF2 (red, $0.030 \pm 0.005 \mathrm{~min}^{-1}$ ) or eEF3 (blue, $0.10 \pm 0.01 \mathrm{~min}^{-1}$ ). Data presented as mean $\pm \mathrm{SEM}$ of $\mathrm{n}=3$. (C) Dissociation of deacylated tRNA from the E site by the fluorescence change of $\mathrm{RNA}^{\mathrm{fMet}}(\mathrm{Flu})$ upon rapidly mixing of $80 \mathrm{~S} 2 \mathrm{C}$ with ternary complexes eEF1A-GTP- $\left[{ }^{14} \mathrm{C}\right] \mathrm{Val}-\mathrm{tRNA}{ }^{\mathrm{Val}}$ in the presence of eEF2 and eEF3 (green), eEF2 (red), eEF3 (blue), or eEF2 and eEF3 without the ternary complex (magenta) in a stopped-flow apparatus. Each trace is an average of 5-7 individual time courses and normalized at 1 for the fluorescence at the start of the reaction. (D) Dissociation of tRNA ${ }^{\mathrm{fMet}}(\mathrm{Flu})$ from $80 \mathrm{~S} 2 \mathrm{C}$ deacylated tRNA incubated with eEF2 and eEF3 (green) or eEF2 (red) for 15 min before adding with ternary complexes. Each trace is an average of 5-7 individual time courses and normalized at 1 for the fluorescence at the start of the reaction. 
A

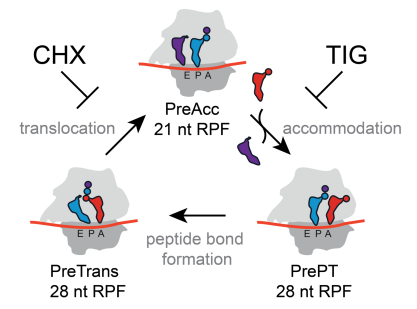

$E$

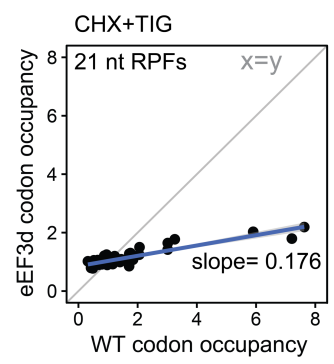

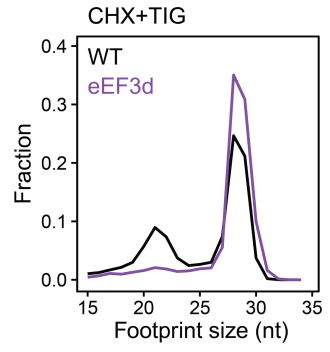

$\mathrm{F}$

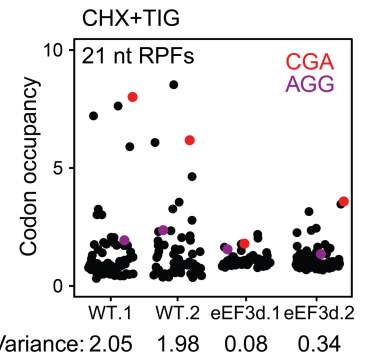

C
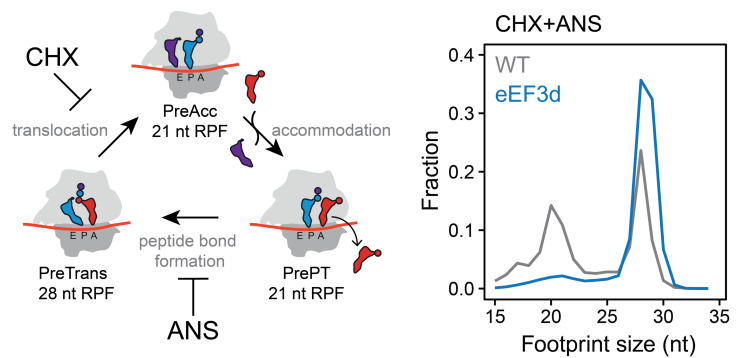

G

$\mathrm{H}$

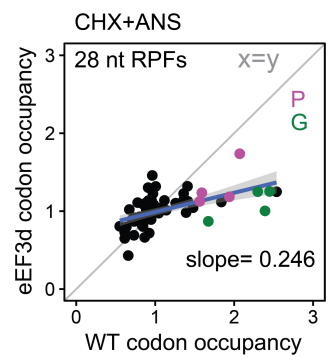

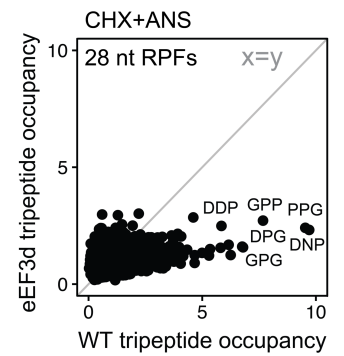

Figure 3. eEF3 depletion perturbs translation elongation transcriptome-wide. (A)

Schematic representation of the eukaryotic elongation cycle with ribosome footprint sizes to illustrate the functional states isolated by $\mathrm{CHX}+\mathrm{TIG}$. PreAcc, pre-accommodation; PrePT, pre-peptide bond formation; PreTrans, pre-translocation. (B) Size distributions of ribosome footprints for WT (black) and eEF3d (purple) cells from libraries prepared with CHX+TIG. (C) Similar to (A), with footprint sizes isolated by CHX+ANS. (D) Similar to (B), from libraries prepared with $\mathrm{CHX}+\mathrm{ANS}$. (E) Scatter plot of codon-specific ribosome occupancies for $21 \mathrm{nt}$ RPFs comparing eEF3d to WT cells from libraries prepared with $\mathrm{CHX}+\mathrm{TIG}$. Trend line (blue) and its slope are shown. The diagonal line indicates the distribution expected for no change. (F) Codon-specific ribosome occupancies for $21 \mathrm{nt}$ RPFs from WT and eEF3d cells (two biological replicates). CGA and AGG codons are shown in red and purple, respectively. Variance for each dataset is indicated. (G) Scatter plot of codon-specific occupancies for $28 \mathrm{nt}$ RPFs comparing eEF3d to WT cells from libraries prepared with $\mathrm{CHX}+\mathrm{ANS}$. Trend line (blue) and its slope are shown. Codons that encode proline and glycine are colored in purple and green, respectively. (H) 28 nt RPFribosome occupancies of 5,771 tripeptide motifs are plotted for WT and eEF3d cells, for motifs more than 100 occurrences in yeast transcriptome. Motif sequences enriched upon eIF5A depletion are labeled. 
A

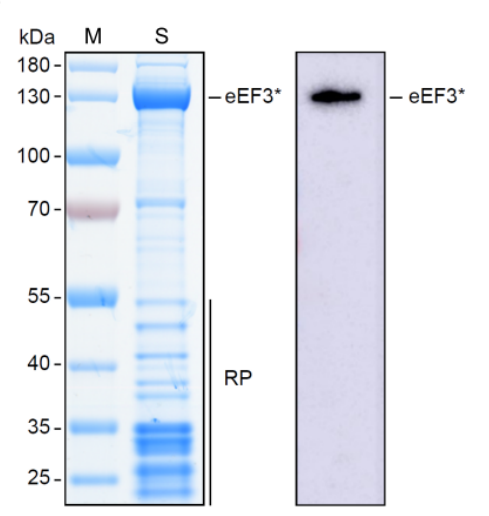

B

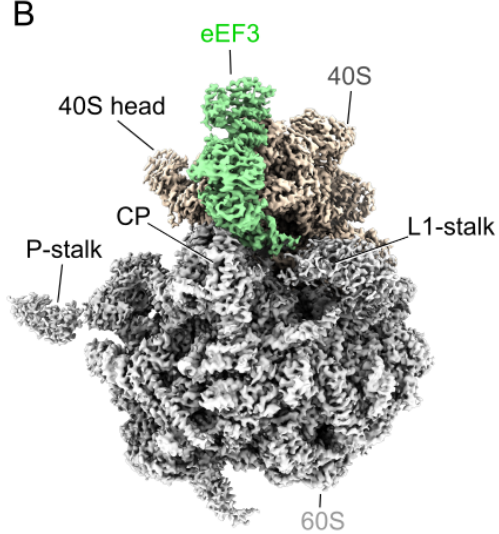

C

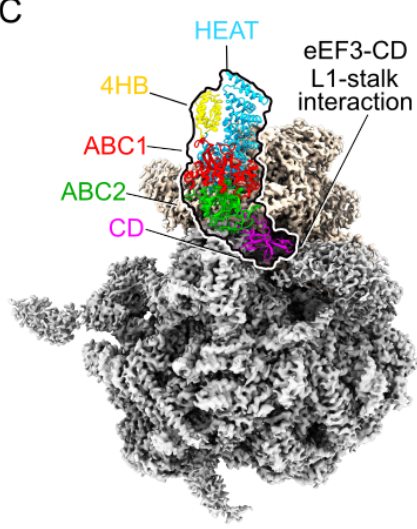

D

E

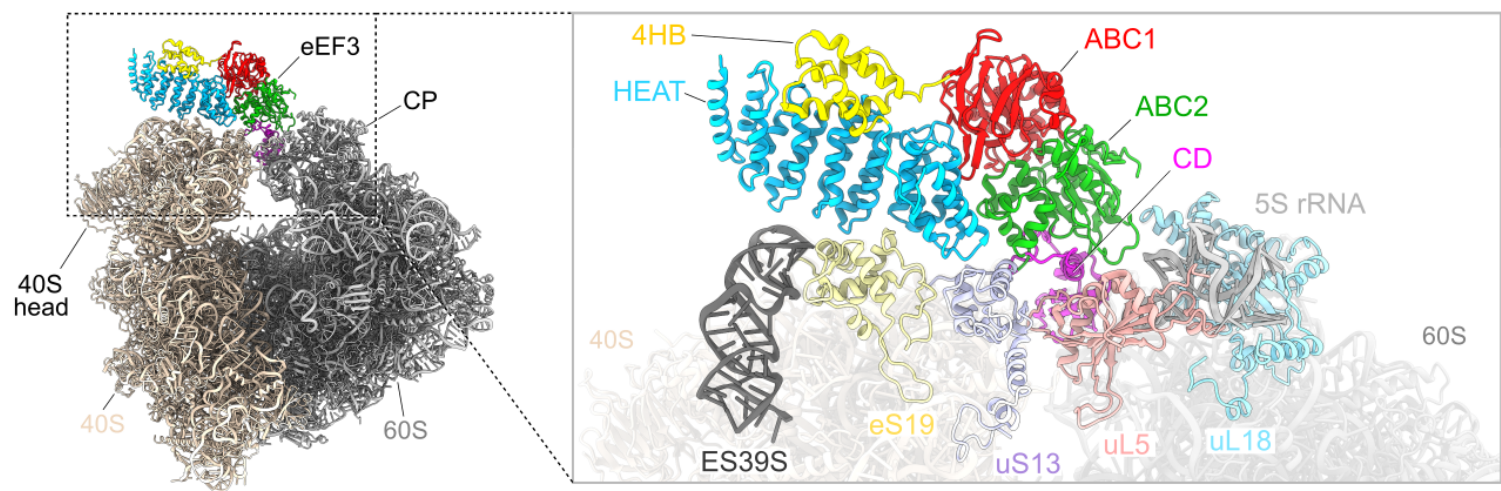

Figure 4. eEF3 in complex with the $S$. cerevisiae $80 \mathrm{~S}$ ribosome. (A) SDS gel and Western Blot of the native pull-out from $S$. cerevisiae TAP-tagged eEF3. eEF3* labels the TEV cleavage product of eEF3-TAP, which still carries the calmodulin binding peptide of the TAP-tag and which is recognized by the antibody. RP, ribosomal proteins. (B) CryoEM reconstruction of the ex vivo eEF3-80S complex with segmented densities for the 60S (grey), the 40S (tan) and eEF3 (pastel green). (C) Fitted eEF3 molecular model colored according to its domain architecture, HEAT (blue), 4HB (yellow), ABC1 (red), ABC2 (green) and CD (magenta). (D) Overview on the eEF3-80S molecular model and (E) zoom illustrating eEF3 and its ribosomal interaction partners. ES39S (dark grey), eS19 (pale yellow), uS13 (pastel violet), uL5 (coral), uL18 (light blue), 5S rRNA (light grey). 
A

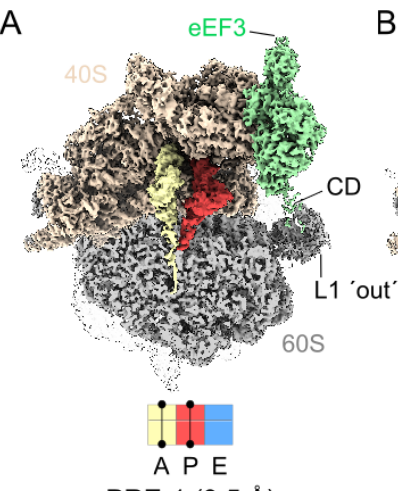

PRE-1 (3.5 ^̊)

$\mathrm{E}$

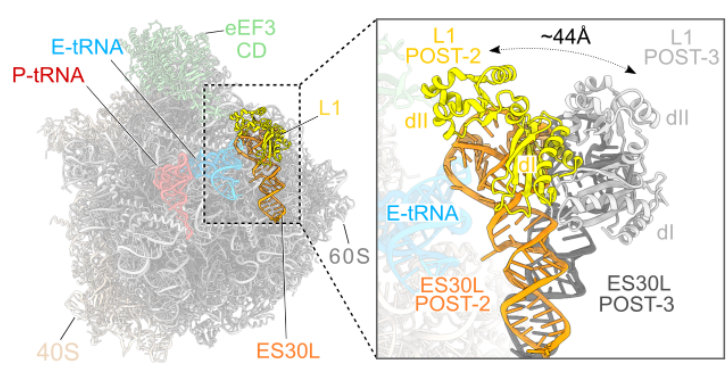

PRE-2 (4.0 Å)

$\mathrm{F}$
C
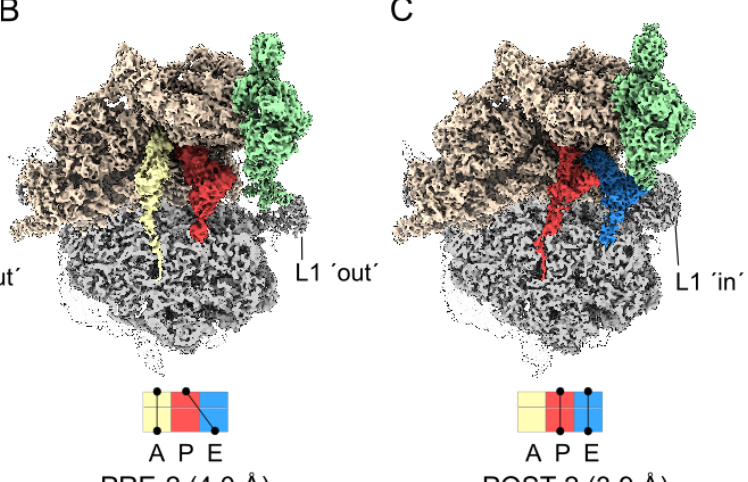

POST-2 (3.9 Å)

G

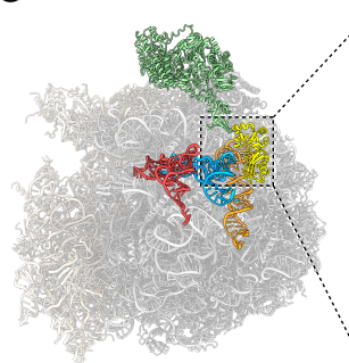

D

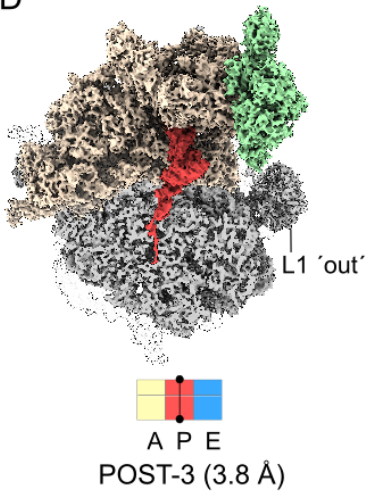

$\mathrm{H}$

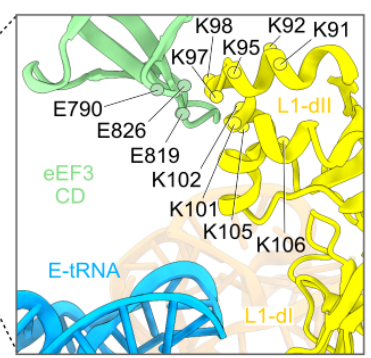

Figure 5. eEF3 bound to a non-rotated $80 S$ ribosome. (A-D) Cryo-EM maps of eEF3 bound to non-rotated ribosomal species with isolated densities for the $60 \mathrm{~S}, 40 \mathrm{~S}$ and eEF3 (colored as in Fig.1), as well as the A- (pale yellow), P- (red) and E-tRNA (blue). The eEF3 ligand is binding the ribosome in the pre-translocational (PRE) state termed as (A) PRE-1 (A/A-, P/P-tRNA) and (B) PRE-2 (A/A-, P/E-tRNA) as well as to the post-translocational (POST) version named (C) POST-2 (P/P-, E/E-tRNA) and (D) POST-3 (P/P-tRNA). (E) Overview of the POST-2 eEF3-80S molecular model highlighting the L1 protein (yellow) and ES30L (orange) and its (F) zoom depicting the magnitude of the L1-stalk movement from the POST-2 state (L1-stalk 'in') to the POST-3 state (L1-stalk 'out'). L1-POST3 (light gray), ES30L-POST3 (dark gray). (G) Different view of the model shown in (E) highlighting eEF3, L1, ES30L as well as the P/P- and E/E-tRNA. (H) Zoom of (G) showing the contact of the eEF3-CD and the L1 protein as well as the distance of both to the EtRNA. The residues are displayed as circles and labeled. 
A

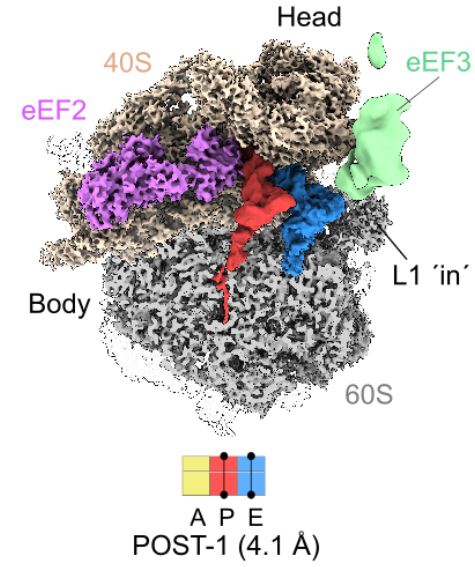

B

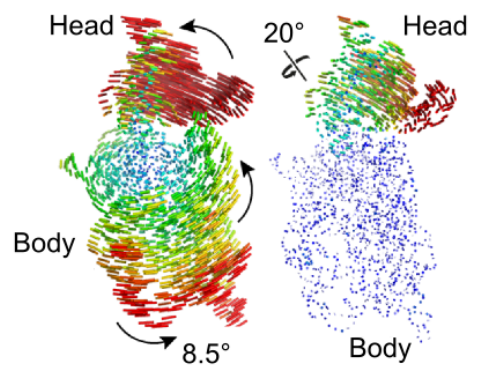

C

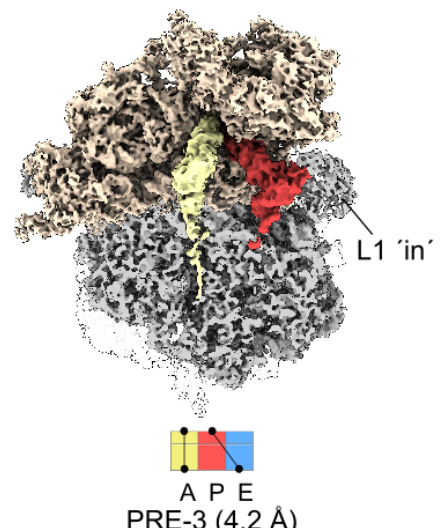

PRE-3 (4.2 Å)

E
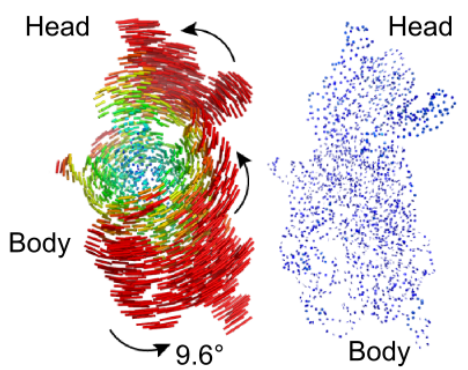

D

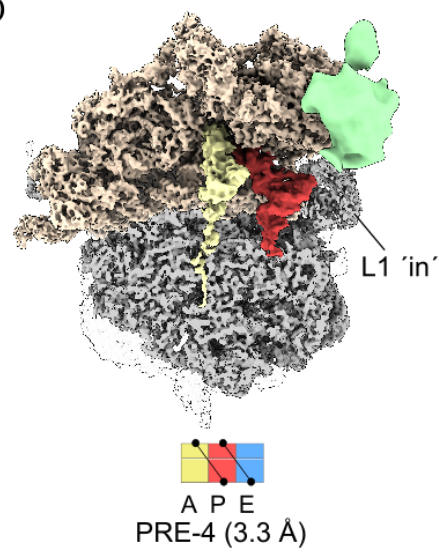

$\mathrm{F}$
Her
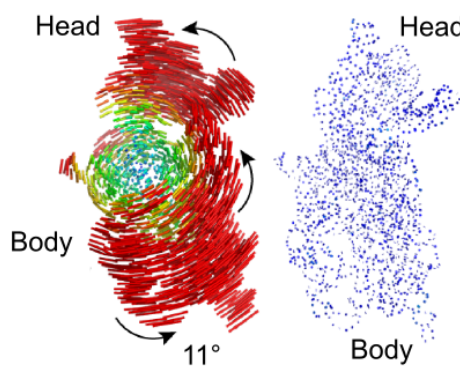

Figure 6. Rotated S. cerevisiae ribosome partially bound by disordered eEF3 ligand.

(A) Cryo-EM reconstruction of the eEF2 bound $80 \mathrm{~S}$ ribosome adopting a rotated POST-1 state bearing a P/P- and E/E-tRNA as well as a disordered eEF3. (B) Subunit rotation and head swivel observed in the $S$. cerevisiae eEF3-80S complex derived from the selected class from the $3 \mathrm{D}$ classification shown in (A). 18S rRNA structures illustrating the degree of rotation of the small subunit relative to the large subunit based alignments of the large subunit from a non-rotated structure reference (PDB ID: 6SNT) (Matsuo et al., 2020). The degree of head swivelling (right side) is illustrated based on alignments of the 18S rRNA body from a non-headed swivel reference structure (PDB ID: 6SNT) (Matsuo et al., 2020). (C-D) $80 \mathrm{~S}$ ribosome in rotated (C) PRE-3 state containing an A/A- and P/E-tRNA and (D) PRE-4 state bearing the hybrid A/P- and P/E-tRNA and bound by the disordered eEF3 ligand. (E-F) Representation of the rotation of the subunit and the 40S-head swivel shown for volumes depicted in (C) and (D), respectively. 


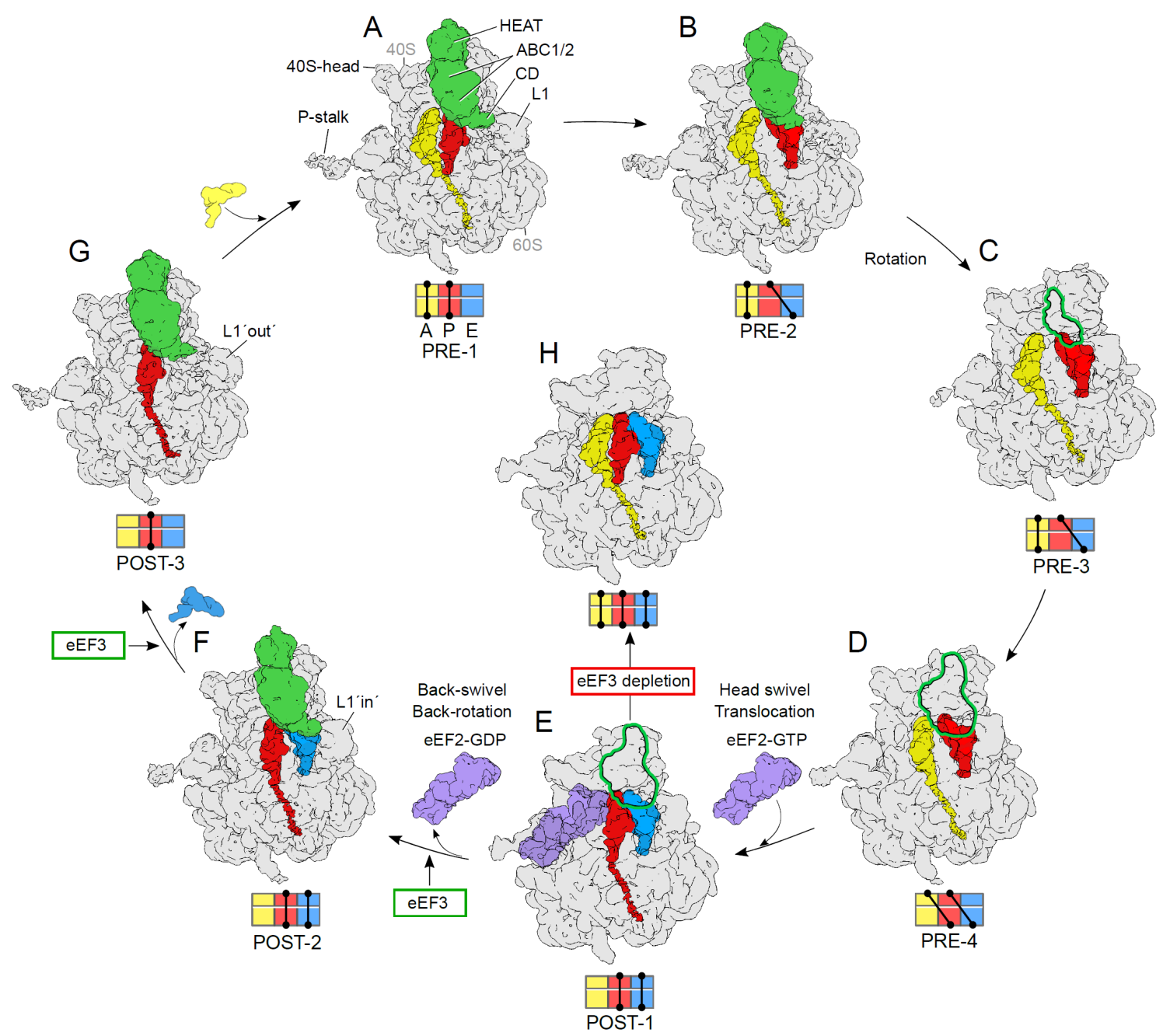

Figure 7. Functional role of eEF3 in the framework of the elongation cycle. (A-B) eEF3 is binding to (A) non-rotated $80 \mathrm{~S}$ in a pre-translocation (PRE) state bearing classical A/Aand P/P-tRNA (PRE-1) as well as to (B) non-rotated state occupied by A/A- and a hybrid P/E-tRNA (PRE-2). (C) Rotation of the ribosomal subunits leads to unstable binding of eEF3 to a ribosome with A/A- and P/E-tRNA (PRE-3) as well as to (D) a fully rotated ribosomal species bearing hybrid A/P- and P/E-tRNAs (PRE-4). (E) Binding of eEF2-GTP facilitates 40S-head swiveling and translocation of the hybrid A/P- and P/E-tRNA into the classical P/P- and E/E-sites (POST-1). (F) After dissociation of eEF2-GDP and the resulting back-swivel and back-rotation of the ribosome, eEF3 stably binds to the nonrotated ribosome with a classical P/P- and E/E-tRNA (POST-2) with L1-stalk in the 'in' position. (G) The eEF3-CD is directly interacting with L1 stabilizing it in an 'out' conformation and enabling the release of the E-site tRNA. eEF3 remains bound to the nonrotated ribosome bearing a P/P-tRNA (POST-3). Transition for state $\mathrm{E}$ to $\mathrm{F}$ and $\mathrm{F}$ to $\mathrm{G}$ are accelerated by eEF3. (H) eEF3-depletion studies suggested a ribosomal species occupied 
by all three tRNAs due to lack of eEF3 overtaking the function of E-site tRNA release. Volumes (A-G) are representing the cryo-EM reconstructions from the eEF3-TAP pullout, whereas volume $(\mathrm{H})$ shows a potential scenario based of the results of the eEF3depletion studies. All maps are filtered to $6 \AA$. The outline shown in (C-E) assigns the disordered eEF3 ligand present in these volumes. 


\section{EXPERIMENTAL PROCEDURES}

\section{E. coli competent cells and growth conditions}

eIF1 was cloned into a pET22b plasmid without affinity tag. eIF1A, eIF5, eIF5A and eIF5 B-397C (lacking 396 amino acids from the C-termini) were cloned into a pGEX-6P1 plasmid with a GST tag. Recombinant proteins were expressed in E. coli BL21(DE3) cells from IPTG-inducible plasmids at $37^{\circ} \mathrm{C}$ for eIF1, eIF5A and eIF5B-397C. eIF1A and eIF5 were expressed from IPTG-inducible plasmids at $16^{\circ} \mathrm{C}$.

\section{S. cerevisiae strain and growth conditions}

The S. cerevisiae cells for ribosomal subunits, eIF2, eEF1A, eEF2 and eEF3 were grown in a $250 \mathrm{~L}$ bioreactor in $1 \mathrm{xYPD}(20 \mathrm{~g} / \mathrm{L}$ peptone, $10 \mathrm{~g} / \mathrm{L}$ yeast extract, $20 \mathrm{~g} / \mathrm{L}$ glucose $)$ at $30^{\circ} \mathrm{C}$. The $S$. cerevisiae cells for eIF3 were grown in a $250 \mathrm{~L}$ bioreactor in CSM-LEUURA media at $30^{\circ} \mathrm{C}$. The S. cerevisiae eEF3-TAP strain (GE Healthcare/Dharmacon, Strain: BY4741, GeneID: 850951) was grown to $\mathrm{OD}_{600}=0.8$ in an InnovaX large-capacity incubator shaker in $1 \mathrm{xYPD}$ at $30^{\circ} \mathrm{C}$.

For ribosome profiling, WT (replicated 2) and yCW35 strains were grown in YPGR (20g/L peptone, $10 \mathrm{~g} / \mathrm{L}$ yeast extract, $20 \mathrm{~g} / \mathrm{L}$ galactose $+20 \mathrm{~g} / \mathrm{L}$ raffinose) overnight at $30^{\circ} \mathrm{C}$, collected by centrifugation, washed, and resuspended in YPD at OD 0.003 and 0.02 , respectively. After $8 \mathrm{hr}$, cells were harvested at OD 0.55 and 0.45 , respectively. Cells were harvested by fast filtration and flash frozen in liquid nitrogen.

\section{METHODS DETAILS}

\section{Buffers}

The following buffers were used for purification of translation components:

Buffer 1. $20 \mathrm{mM}$ Hepes/KOH pH 7.5, $100 \mathrm{mM} \mathrm{KOAc,} 2.5 \mathrm{mM} \mathrm{Mg}(\mathrm{OAc})_{2}, 1 \mathrm{mg} / \mathrm{ml}$ heparin sodium salt, $2 \mathrm{mM}$ DTT.

Buffer 2. $20 \mathrm{mM}$ Hepes/KOH pH 7.5, $100 \mathrm{mM} \mathrm{KOAc,} 400 \mathrm{mM} \mathrm{KCl,} 2.5 \mathrm{mM} \mathrm{Mg}(\mathrm{OAc})_{2}$, $1 \mathrm{M}$ sucrose, $2 \mathrm{mM}$ DTT.

Buffer 3. 20 mM Hepes/KOH pH 7.5, 100 mM KOAc, 400 mM KCl, 2.5 mM Mg(OAc)2, $1 \mathrm{mg} / \mathrm{ml}$ heparin sodium salt, $2 \mathrm{mM}$ DTT. 
Buffer 4. 50 mM HEPES-KOH pH 7.5, $500 \mathrm{mM} \mathrm{KCl,} 2 \mathrm{mM} \mathrm{MgCl} 2,2 \mathrm{mM}$ DTT.

Buffer 5. 50 mM HEPES-KOH pH 7.5, $500 \mathrm{mM} \mathrm{KCl,} 5 \mathrm{mM} \mathrm{MgCl} 2,2 \mathrm{mM}$ DTT, $0.1 \mathrm{mM}$ EDTA, 5\% sucrose.

Buffer 6. 50 mM HEPES-KOH pH 7.5, $500 \mathrm{mM} \mathrm{KCl,} 5 \mathrm{mM} \mathrm{MgCl}_{2}, 2 \mathrm{mM}$ DTT, $0.1 \mathrm{mM}$ EDTA, 30\% sucrose.

Buffer 7. $50 \mathrm{mM}$ HEPES-KOH, $100 \mathrm{mM} \mathrm{KCl,} 250 \mathrm{mM}$ sucrose, $2.5 \mathrm{mM} \mathrm{MgCl}$, $2 \mathrm{mM}$ DTT.

Buffer 8. $20 \mathrm{mM}$ HEPES-NaOH pH 7.5, $150 \mathrm{mM} \mathrm{NaCl}, 5 \%$ glycerol, $4 \mathrm{mM}$ ßmercaptoethanol (ß-me).

Buffer 9. Same as buffer 8 with $1 \mathrm{M} \mathrm{NaCl}$.

Buffer 10. Same as buffer 8 without $\mathrm{NaCl}$ and glycerol.

Buffer 11. 20 mM HEPES-KOH pH 7.5, 200 mM KCl, 2 mM DTT.

Buffer 12. 20 mM HEPES-NaOH pH 7.5, 500 mM NaCl, 5\% glycerol, 4 mM ß-me.

Buffer 13. 50 mM HEPES-NaOH pH 7.5, 400 mM NaCl, 5\% glycerol, 4 mM ß-me.

Buffer 14. 20 mM HEPES-KOH pH 7.5, 500 mM KCl, 10\% glycerol, 3 mM ß-me.

Buffer 15. $20 \mathrm{mM}$ 3-(N-morpholino)propane sulfonic acid (MOPS)-KOH pH 6.7, $500 \mathrm{mM}$ $\mathrm{KCl}, 10 \%$ glycerol, $3 \mathrm{mM} ß-\mathrm{me}$.

Buffer 16. 20 mM HEPES-NaOH pH 7.5, 500 mM NaCl, 30 mM L-glutathione reduced, 5\% glycerol, 4 mM ß-me.

Buffer 17. 20 mM HEPES-KOH pH 7.5, 100 mM KCl, 2 mM DTT.

Buffer 18. 20 mM HEPES-KOH pH 7.5, 200 mM KCl, 2 mM DTT.

Buffer 19. 20 mM HEPES-KOH pH 7.5, 100 mM KCl, 5\% glycerol, 2 mM DTT.

Buffer 20. 20 mM HEPES-KOH pH 7.5, 100 mM KCl, 5\% glycerol, 2 mM DTT.

Buffer 21. 20 mM HEPES-NaOH pH 7.5, $1 \mathrm{M} \mathrm{NaCl,} \mathrm{5 \%} \mathrm{glycerol,} 4$ mM ß-me.

Buffer 22. $50 \mathrm{mM}$ Tris- $\mathrm{HCl} \mathrm{pH} 7.5,5 \mathrm{mM} \mathrm{MgCl}_{2}, 50 \mathrm{mM} \mathrm{NH} 4 \mathrm{Cl}, 0.2 \mathrm{mM} \mathrm{PMSF}, 10 \%$ glycerol, 0.1 mM EDTA pH 8.0, 1 mM DTT.

Buffer 23. 20 mM Tris-HCl pH 7.5, 50 mM KCl, 0.1 mM EDTA pH 8.0, 0.2 mM PMSF, 25\% glycerol, $1 \mathrm{mM}$ DTT.

Buffer 24. 20 mM Tris- $\mathrm{HCl}$ pH 7.5, 500 mM KCl, 0.1 mM EDTA pH 8.0, 0.2 mM PMSF, $25 \%$ glycerol, 1 mM DTT. 
Buffer 25. 20 mM Tris-HCl pH 7.5, 0.1 mM EDTA pH 8.0, 200 mM KCl, 25\% glycerol, $1 \mathrm{mM}$ DTT.

Buffer 26. 20 mM HEPES-KOH pH 7.5, $500 \mathrm{mM} \mathrm{KCl,} 20 \mathrm{mM}$ imidazole, 10\% glycerol, 2 mM ß-me.

Buffer 27. 20 mM HEPES- KOH pH 7.5, $100 \mathrm{mM} \mathrm{KCl,} 250 \mathrm{mM}$ imidazole, 10\% glycerol, 2 mM ß-me.

Buffer 28. 20 mM HEPES- KOH pH 7.5, 100 mM KCl, 10\% glycerol, 2 mM DTT.

Buffer 29. $40 \mathrm{mM}$ Tris- $\mathrm{HCl} \mathrm{pH}$ 7.5, $15 \mathrm{mM} \mathrm{MgCl}, 2 \mathrm{mM}$ spermidine, $10 \mathrm{mM} \mathrm{NaCl}$.

\section{Preparation of ribosomal subunits, initiation and elongation factors}

$80 \mathrm{~S}$ ribosomal subunits, eIF2, eIF3, eEF2 and eEF3 were prepared from $S$. cerevisiae as described previously (Acker et al., 2007; Algire et al., 2002; Jorgensen et al., 2002; Pavitt et al., 1998; Phan et al., 2001; Sasikumar and Kinzy, 2014).

For $80 \mathrm{~S}$ ribosomal subunits purification, cells were harvested in mid-log phase and resuspended in $1 \mathrm{ml} / \mathrm{g}$ of cells in lysis buffer 1 . Cell pellets frozen in liquid nitrogen were ground using an ultra-centrifugal mill according to the CryoMill protocol $\left(\operatorname{Retsch}^{\odot}\right)$. The lysate was thawed at $4^{\circ} \mathrm{C}, 100 \mu \mathrm{DN}$ Dase and one EDTA-free protease inhibitor tablet was added and incubated at $4^{\circ} \mathrm{C}$ for $30 \mathrm{~min}$. The thawed lysate was clarified by centrifugation at $13,000 \mathrm{rpm}$ at $4^{\circ} \mathrm{C}$ for $30 \mathrm{~min}$. The salt concentration of the supernatant was increased to $500 \mathrm{mM} \mathrm{KCl}$ and was then filtered using $1 \mu \mathrm{m}$ glass fiber filters. Ribosomes in the supernatant were collected in buffer 2 at $45,000 \mathrm{rpm}$ at $4^{\circ} \mathrm{C}$ for $2 \mathrm{~h}$ in a Ti45 rotor. Ribosomal pellets were resuspended in resuspension buffer 3 and were incubated on ice for $15 \mathrm{~min}$. Ribosomes were collected once more through buffer 2 at $100,000 \mathrm{rpm}$ at $4^{\circ} \mathrm{C}$ for $30 \mathrm{~min}$ in a MLA 130 rotor. The pellets were resuspended in buffer 4, incubated on ice for $15 \mathrm{~min}$ with $1 \mathrm{mM}$ puromycin and then $10 \mathrm{~min}$ at $37^{\circ} \mathrm{C}$. The sample was loaded on a $5 \%-30 \%$ sucrose gradient (buffer $5 \& 6$ ) and centrifuged at $25,000 \mathrm{rpm}$ at $4^{\circ} \mathrm{C}$ for $16 \mathrm{~h}$. The 40S and 60S subunits were collected from gradient fractionation, were exchanged separately into buffer 7 , concentrated and stored at $-80^{\circ} \mathrm{C}$ after being flash frozen in liquid nitrogen.

eIF1 cell pellets was resuspended in buffer 8, lysed in the presence of DNase and protease inhibitor tablet, and purified by HiTrap SP cation exchange chromatography with a linear 
gradient from $0 \%$ to $100 \%$ buffer 9 over $60 \mathrm{~mL}$ after equilibrating the column and loading eIF1 with buffer 8 . The fractions containing eIF1 were pooled, and diluted to decrease the salt concentration to $150 \mathrm{mM}$ with buffer 10. Next, eIF1 was purified by HiTrap Heparin chromatography with a linear gradient from $0 \%$ to $100 \%$ buffer 9 over $60 \mathrm{~mL}$ after equilibrating the column and loading eIF1 with buffer 8 . The fractions containing eIF1 were pooled, concentrated and further purification was attained by size-exclusion chromatography on a HiLoad 26/600 Superdex 75pg column with buffer 11 . The purified eIF1 protein was concentrated and stored in buffer 5 at $-80^{\circ} \mathrm{C}$.

eIF1A, eIF5, eIF5A and eIF5 B-397C cell pellets were resuspended in buffer 12, 13, 14 and 15, respectively, and lysed in the presence of DNase and protease inhibitor tablet. All GST-tagged proteins were purified by GSTrap column with $100 \%$ buffer 16 . After cleavage of the fusion protein with PreScission protease ( $1 \mu \mathrm{M}$ final), further purification was attained by size-exclusion chromatography on a HiLoad 26/600 Superdex 75 pg (eIF1A with buffer 17, eIF5 with buffer 18, eIF5A with buffer 19) and HiLoad 26/600 Superdex 200 pg (eIF5B-397C with buffer 20) column. For eIF1A, before size-exclusion chromatography, an additional step of a Resource Q anion exchange chromatography was included after protease cleavage and protein was eluted with $0 \%$ to $100 \%$ buffer 21 over $60 \mathrm{~mL}$. The purified proteins were concentrated and stored at $-80^{\circ} \mathrm{C}$.

eEF1A was purified from $S$. cerevisiae. eEF1A cell pellets was resuspended in buffer 22, lysed using an ultra-centrifugal mill, and the lysate was loaded on a tandom HiTrap Q anion exchange and Hi Trap SP cation exchange column pre-equilibrated in buffer 23. eEF1A was eluted from HiTrap SP with $0 \%$ to $100 \%$ buffer 24 over $30 \mathrm{~mL}$. Further purification was attained by size-exclusion chromatography on a HiLoad 26/600 Superdex 200 pg with buffer 25 . The purified eEF1A protein was concentrated and stored at $-80^{\circ} \mathrm{C}$.

\section{In vitro hypusination of eIF5A}

eIF5A hypusination enzymes deoxyhypusine synthase (Dys1) and deoxyhypusine hydroxylase (Lia1) were co-expressed in Escherichia coli BL21(DE3) cells from IPTGinducible $\mathrm{pQLinkH}$ plasmid with a His6 tag at $37^{\circ} \mathrm{C}$. Cell pellet was resuspended in buffer 26 , lysed in the presence of DNase and protease inhibitor tablet. The protein was purified by Protino Ni-IDA 2000 affinity chromatography with buffer 27, after equilibrating the 
column and loading the protein with buffer 26 . The eluted protein was dialyzed in buffer 28 , concentrated and stored at $-80^{\circ} \mathrm{C}$. The in vitro hypusination of eIF5A was performed as described previously (Park et al., 2011; Wolff et al., 2011).

\section{Preparation of tRNAs and mRNAs}

Initiator tRNA (tRNA ${ }_{i}{ }^{\mathrm{Met}}$ ) was prepared by in vitro transcription using $\mathrm{T} 7$ polymerase from a plasmid containing a 92 nucleotides-long DNA with the T7 promoter (underlined) and the initiator tRNA sequence purchased from Eurofins.

\section{5'TAATACGACTCACTATAAGCGCCGTGGCGCAGTGGAAGCGCGCAGGGCTC} ATAACCCTGATGTCCTCGGATCGAAACCGAGCGGCGCTACCA3'

The DNA was amplified using forward and reverse primers and the amplified product was in vitro transcribed in buffer 29 with $10 \mathrm{mM}$ DTT, $3 \mathrm{mM} \mathrm{NTP} \mathrm{mix,} 0.005 \mathrm{U} / \mu \mathrm{L}$ inorganic Pyrophosphate (PPase), $0.1 \mathrm{U} / \mu \mathrm{L}$ RNase inhibitor and $0.05 \mathrm{U} / \mu \mathrm{L}$ T7 RNA-polymerase for 4 hours at $37^{\circ} \mathrm{C}$. Aminoacylation and purification of $\left[{ }^{3} \mathrm{H}\right] \mathrm{Met}-\mathrm{tRNA} \mathrm{i}_{\mathrm{i}}{ }^{\mathrm{Met}}$ and $\left[{ }^{3} \mathrm{H}\right] \mathrm{Met}-$ tRNA $_{i}{ }^{M e t}$ (Flu) were performed as described previously (Milon et al., 2007; Rodnina et al., 1994).

The elongator tRNAs $\left[{ }^{14} \mathrm{C}\right]$ Val-tRNA ${ }^{\mathrm{Val}},\left[{ }^{14} \mathrm{C}\right] \mathrm{Phe}^{-t R N A}{ }^{\text {Phe }}$ and Phe-tRNA ${ }^{\text {Phe }}$ were prepared as described in (Rodnina et al., 1994). The unstructured 5' UTR mRNA Met-PheVal and mRNA Met-Val-Phe were purchased from IBA (mRNA sequence in STAR Methods).

\section{Preparation of initiation and ternary complexes}

$80 \mathrm{~S}$ initiation complexes were prepared by incubating $8 \mu \mathrm{M}$ of eIF2 with $1 \mathrm{mM}$ GTP and $4 \mu \mathrm{M}\left[{ }^{3} \mathrm{H}\right]$ Met-tRNA ${ }_{i}{ }^{\text {Met }}$ or $\left[{ }^{3} \mathrm{H}\right]$ Met-tRNA ${ }_{i}{ }^{\text {Met }}$ (Flu) in YT buffer (30 mM HEPES-KOH $\mathrm{pH} 7.5,100 \mathrm{mM} \mathrm{KOAc,} 3 \mathrm{mM} \mathrm{MgCl} 2)$ at $26^{\circ} \mathrm{C}$ for $15 \mathrm{~min}$ to form the ternary complex. 2 $\mu \mathrm{M}$ 40S, $10 \mu \mathrm{M}$ mRNA (uncapped mRNA with unstructured 5' UTR that alleviates the requirement of eIF4 (Acker et al., 2007)), $10 \mu \mathrm{M}$ eIF-mix (mixture of initiation factors eIF1, eIF1A, eIF3, eIF5), 2 mM DTT, $0.25 \mathrm{mM}$ spermidine and $1 \mathrm{mM}$ GTP were incubated for $5 \mathrm{~min}$ at $26^{\circ} \mathrm{C}$ before adding $3 \mu \mathrm{M} 60 \mathrm{~S}$ subunits and $6 \mu \mathrm{M}$ eIF5B. After incubation, ternary complex was added to the ribosome mixture and the $\mathrm{MgCl}_{2}$ was adjusted to a final concentration of $9 \mathrm{mM}$ before layering on sucrose cushion. ICs were purified by 
ultracentrifugation through a 1.1 M sucrose cushion in YT9 buffer (30 mM Hepes-KOH $\mathrm{pH}$ 7.5, $100 \mathrm{mM} \mathrm{KoAc,} 9 \mathrm{mM} \mathrm{MgCl}$ ), and pellets were dissolved in YT9 buffer.

Ternary complexes eEF1A-GTP- $\left[{ }^{14} \mathrm{C}\right]$ Phe-tRNA ${ }^{\text {Phe }}$ and eEF1A-GTP- $\left[{ }^{14} \mathrm{C}\right]$ Val-tRNA Val were prepared by incubating $1 \mu \mathrm{M}$ eEF1A, $0.1 \mu \mathrm{M}$ eEF1B $\alpha, 3 \mathrm{mM}$ PEP, $1 \%$ PK, $1 \mathrm{mM}$ DTT, $0.5 \mathrm{mM}$ GTP in YT buffer for $15 \mathrm{~min}$ at $26^{\circ} \mathrm{C} .0 .2 \mu \mathrm{M}\left[{ }^{14} \mathrm{C}\right] \mathrm{Phe}$-tRNA ${ }^{\text {Phe }}$ or $\left[{ }^{14} \mathrm{C}\right]$ ValtRNA $^{\text {Val }}$ (5 eEF1A:1 aa-tRNA) was added and incubated for additional $5 \mathrm{~min}$ at $26^{\circ} \mathrm{C}$, followed by addition of $2 \mu \mathrm{M}$ modified eIF5A.

\section{Rapid kinetics}

Peptide bond formation assay were performed by rapidly mixing initiation complexes (1 $\mu \mathrm{M})$ with the respective ternary complexes as indicated $(0.2 \mu \mathrm{M})$ and eEF1A $(1 \mu \mathrm{M})$ in a quench-flow apparatus at $26^{\circ} \mathrm{C}$. After the desired incubation times the reactions were quenched by adding $\mathrm{KOH}$ to a final concentration of $0.5 \mathrm{M}$. Peptides were released by alkaline hydrolysis for $45 \mathrm{~min}$ at $37^{\circ} \mathrm{C}$. After neutralization with acetic acid, the products were analyzed by HPLC (LiChrospher100 RP-8 HPLC column, Merck). To form tripeptides, MetPhe-tRNA ${ }^{\text {Phe }}$ pretranslocation complexes $(0.35 \mu \mathrm{M})$ were rapidly mix with the respective ternary complex $(0.7 \mu \mathrm{M})$ containing $1 \mu \mathrm{M}$ eEF2 and $4 \mu \mathrm{M}$ eEF3.

Deacylated tRNA release from the E site were performed by rapidly mixing initiation complexes prepared using $\left[{ }^{3} \mathrm{H}\right] \mathrm{Met}-\mathrm{RNA}_{i}{ }^{\mathrm{Met}}(\mathrm{Flu})$ with the respective ternary complexes as indicated $(0.2 \mu \mathrm{M})$ and eEF1A $(1 \mu \mathrm{M})$ in a stopped-flow apparatus (Applied Photophysics) at $26^{\circ} \mathrm{C}$. Fluorescein fluorophore was excited at $463 \mathrm{~nm}$ and emission was measured after passing through KV500 long-pass filters (Schott). Experiments were performed by rapidly mixing equal volumes of reactants and monitoring the time courses of fluorescence changes. Time courses depicted in the figures were obtained by averaging 5-7 individual traces.

\section{Time-resolved Pmn assay}

To monitor translocation for the MetPhe-tRNA ${ }^{\text {Phe }}$ PRE complex, PRE complex $(0.35 \mu \mathrm{M})$ was rapidly mixed with Pmn $(2 \mathrm{mM})$, and/or eEF2 $(0.8 \mu \mathrm{M})$ and/or eEF3 $(2 \mu \mathrm{M})$ in YT buffer at $26^{\circ} \mathrm{C}$. Control experiments were carried out with POST complexes prepared by incubating PRE complexes with eEF2 and/or eEF3. POST complexes with MetPhe- 
$\operatorname{tRNA}^{\text {Phe }}(0.35 \mu \mathrm{M})$ in the P site were rapidly mixed with Pmn $(2 \mathrm{mM})$ in a quench-flow apparatus. The reaction was quenched with $\mathrm{KOH}(0.5 \mathrm{M})$ and the peptides were released by alkaline hydrolysis for $45 \mathrm{~min}$ at $37^{\circ} \mathrm{C}$, analyzed by reversed-phase HPLC (LiChrospher $100 \mathrm{RP}-8$, Merck), and quantified by double-label $\left[{ }^{3} \mathrm{H}\right] \mathrm{Met}-\left[{ }^{14} \mathrm{C}\right] \mathrm{Phe}$ radioactivity counting (Wohlgemuth et al., 2008).

\section{Preparation of libraries for yeast ribosome footprints}

Cell pellets were ground with $1 \mathrm{~mL}$ yeast footprint lysis buffer $[20 \mathrm{mM}$ Tris- $\mathrm{Cl}(\mathrm{pH} 8.0)$, $140 \mathrm{mM} \mathrm{KCl}, 1.5 \mathrm{mM} \mathrm{MgCl} 2,1 \%$ Triton X-100 with specified elongation inhibitors] in a Spex 6870 freezer mill. Elongation inhibitors (CHX, ANS and TIG) were used at $0.1 \mathrm{~g} / \mathrm{L}$. Lysed cell pellets were diluted to $\sim 23 \mathrm{~mL}$ in yeast footprint lysis buffer containing specified antibiotics and clarified by centrifugation. The resultant supernatant was layered on a sucrose cushion [20 mM Tris-Cl (pH8.0), $150 \mathrm{mM} \mathrm{KCl,} 5 \mathrm{mM} \mathrm{MgCl} 2,0.5 \mathrm{mM}$ DTT, $1 \mathrm{M}$ sucrose] to pellet polysomes in a Type 70Ti rotor (Beckman Coulter) (60,000 rpm for 106 $\mathrm{mi}$ ). Ribosome pellets were gently resuspended in $1 \mathrm{~mL}$ footprint lysis buffer (without elongation inhibitors). $400 \mu \mathrm{g}$ of isolated polysomes in $350 \mu \mathrm{L}$ of footprint lysis buffer (without elongation inhibitors) were treated with 500 units of RNaseI (Ambion) for $1 \mathrm{hr}$ at $25^{\circ} \mathrm{C}$. Monosomes were isolated by sucrose gradients $(10-50 \%)$. The extracted RNA was size-selected from 15\% denaturing PAGE gels, cutting between 15-34 nt. An oligonucleotide adapter was ligated to the 3' end of isolated fragments. After ribosomal RNA depletion using RiboZero (Illumina), reverse transcription using SuperScript III reverse transcriptase (Thermo Fisher Scientific), circularization using CirLigase I (Lugicen) and PCR amplification. Libraries were sequenced on a HiSeq2500 machine at facilities at the Johns Hopkins Institute of Genetic Medicine.

\section{Tandem affinity purification of the eEF3-80S complex}

The eEF3 in vivo pull-out was performed using DynabeadsR CM-270 Epoxy (Invitrogen) with yeast strain expressing a C-terminally TAP-tagged eEF3 (Strain: BY4741, Genotype:

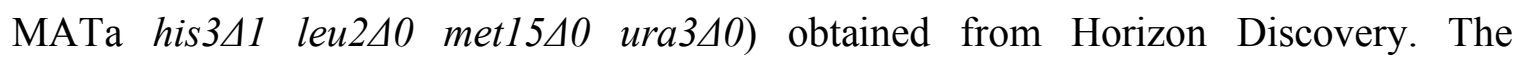
purification was essentially performed as described previously (Schmidt et al., 2016). Cultures were harvested at log phase, lysed by glass bead disruption and incubated with 
IgG-coupled magnetic beads with slow tilt rotation for $1 \mathrm{~h}$ at $4{ }^{\circ} \mathrm{C}$. The elution was done by addition of AcTEV Protease for $3 \mathrm{~h}$ at $17^{\circ} \mathrm{C}$ in elution buffer 22 (20 mM HEPES (pH 7.4). $100 \mathrm{mM}$ KOAc, $10 \mathrm{mM} \mathrm{Mg(OAc)}$, $1 \mathrm{mM}$ DTT) containing $1 \mathrm{mM}$ AMP-PNP (Sigma) final concentration.

\section{Sample and grid preparation}

The final complex was crosslinked with $0.02 \%$ glutaraldehyde for $20 \mathrm{~min}$ on ice, and the reaction was subsequently quenched with $25 \mathrm{mM}$ Tris- $\mathrm{HCl} \mathrm{pH}$ 7.5. DDM (Sigma) was added to the sample to a final concentration of $0.01 \%(\mathrm{v} / \mathrm{v})$. For grid preparation, $5 \mu \mathrm{L}(8$ $\mathrm{A}_{260} / \mathrm{mL}$ ) of the freshly prepared crosslinked complex was applied to $2 \mathrm{~nm}$ precoated Quantifoil R3/3 holey carbon supported grids and vitrified using a Vitrobot Mark IV (FEI, Netherlands).

\section{Cryo-electron microscopy and single-particle reconstruction}

Data collection was performed on a FEI Titan Krios transmission electron microscope (TEM) (Thermo Fisher) equipped with a Falcon II direct electron detector (FEI). Data were collected at $300 \mathrm{kV}$ with a total dose of $25 \mathrm{e}^{-} / \AA^{2}$ fractionated over 10 frames with a pixel size of $1.084 \AA$ pixel and a target defocus range of -1.3 to $-2.8 \mu \mathrm{m}$ using the EPU software (Thermo Fisher). The raw movie frames were summed and corrected for drift and beaminduced motion at the micrograph level using MotionCor2 (Zheng et al., 2017). The resolution range of each micrograph and the contrast transfer function (CTF) were estimated with Gctf (Zhang, 2016). A total of 22,856 micrographs were collected. After manual inspection, 18,016 micrographs were used for automated particle picking with Gautomatch (http://www.mrc-lmb.cam.ac.uk/kzhang/) resulting in 530,517 initial particles, of which 211,727 were selected for further processing upon 2D classification in RELION-2.1 (Kimanius et al., 2016). After initial alignment with a vacant 80S reference, the 211,727 particles (defined as 100\%) were 3D classified into 8 classes (Figure S5). Class 1 and 2 (joined to group I) contained 70,780 particles $(\sim 33 \%)$ and displayed density for the eEF3-80S complex but had mixed tRNAs with varying occupancy as well as a dynamic L1-stalk. Class 3 (group II) showed a density for eEF2, whereas class 4 and 5 (group III) revealed rotated ribosomal species with hybrid tRNAs. Both groups (group I and II) 
showed weak density for eEF3. Class 6, 7 and 8 (group IV) ( 31\%) had low resolution ribosomal species with biased orientation, however all of them showed an extra density for eEF3 (not shown). To increase the resolution of the eEF3 ligand and separate it from low resolution eEF3 species, group I was subjected to focused sorting using a mask encompassing the eEF3 ligand (Figure S5). The resulting class 3 containing 45,032 particles $(\sim 21 \%)$ was 3D and CTF refined using RELION-3.0 (Zivanov et al., 2018). The final refined volume was furthermore subjected to multi-body (MB) refinement, for which three masks were used: the first one encompassed one portion of eEF3 (ABC1, ABC2 and $\mathrm{CD}$; residue range 420-976, MB-1) and the 60S, the second mask covered the $40 \mathrm{~S}$ body (MB-2), and the third mask included the remaining part of eEF3 (HEAT and 4HB; residue range 1-419, MB3-3) as well as the 40S head (Figure $\mathbf{S 5}$ and S6F). The final reconstructions were corrected for the modulation transfer function of the Falcon 2 detector and sharpened by applying a negative B-factor estimated by RELION-3.0 (Zivanov et al., 2018). For the sharpening, a mask for the whole eEF3-80S complex was applied resulting in a final reconstruction of $3.3 \AA$ (Figure S5 and S6A). The same was done for each part of the multi-body refined volumes, which provided a resolution of $3.2 \AA$ for the $60 \mathrm{~S}-\mathrm{eEF} 3$ (ABC1/2, CD), $3.3 \AA$ for the $40 \mathrm{~S}$ body and $3.5 \AA$ for the $40 \mathrm{~S}$ head-eEF3 (HEAT, 4HB) (Figure S6E). To obtain a stoichiometric tRNA occupancy as well as defined position of the L1-stalk interacting with the EF3-CD, group I was also subjected to further sorting into four classes using a flat cylinder mask encompassing the relevant regions (tRNAs, L1-stalk and the eEF3-CD) (Figure S5). Each class was subsequently subjected to 3D and CTF refinement and a final postprocessing step. All the resulting classes were in an unrotated state bearing an A/A-, P/P- (Ia, 3.7 §), P/P- (Ib, $3.8 \AA$ ) , A/A-, P/E- (Ic, $4.0 \AA$ ) and a P/Pand E/E-site (Id, $3.9 \AA$ ) tRNAs. Particles of group II were extracted and subjected to CTF and $3 \mathrm{D}$ refinement resulting in a final cryo-EM reconstruction at $4.1 \AA$. For distinct tRNA occupancy of each $80 \mathrm{~S}$ ribosome, group III $(66,551$ particles) was further $3 \mathrm{D}$ classified into two classes and each class was subsequently 3D and CTF refined. Both resulted in a ribosomal species with a disordered eEF3 (IIIa, IIIb), which was visible after low pass filtering of the map. IIIa showed a rotated-1 state containing A/A- and P/E-site tRNAs with a final resolution of $4.2 \AA$. IIIb presented a classical fully rotated state (rotated-2) bearing a hybrid A/P- and P/E-site tRNA at $3.8 \AA$ resolution. The resolutions for all volumes were 
estimated using the "gold standard" criterion $(\mathrm{FSC}=0.143)$ (Scheres, 2012). Localresolution estimation and local filtering of the final volumes was done using Relion-3.0 (Figure S6B-C, F-G).

\section{Molecular modelling}

The eEF3 model was based on the crystal structure of eEF3 in complex with ADP (PDB: 2IW3) with a $2.4 \AA$ resolution (Andersen et al., 2006). The existing ribosome-bound eEF3 model (PDB: 2IX3) was used as a help for the rough fitting of the separate eEF3 domains (HEAT, $4 \mathrm{HB}, \mathrm{ABC} 1, \mathrm{ABC} 2$ and $\mathrm{CD}$ ) of the crystal structure into the density (Andersen et al., 2006). The single domains were fitted with UCSF Chimera 1.12.1 (Pettersen et al., 2004) via the command 'fit in map' and manually adjusted with Coot version 0.8.9.2 (Emsley and Cowtan, 2004). For the manual adjustment, the multi-body refined maps were used for the corresponding parts of the eEF3 model (MB-1 for ABC1, ABC2 and the CD; MB-3 for HEAT and 4HB). The model of the $S$. cerevisiae 80 S ribosome was derived from PDB ID 6S47 (Kasari et al., 2019b) and the model for the L1 protein from the PDB ID 2 NOQ (Schuler et al., 2006). The proteins of the 40S and 60S were fitted separately into locally-filtered electron density maps using UCSF Chimera (Pettersen et al., 2004). The rRNA was fitted domain-wise in Coot (Emsley and Cowtan, 2004). Afterwards manual adjustments were applied to all fitted molecular models using Coot. The combined molecular model (proteins+rRNA) was refined using the phenix.real_space_refine command of phenix version 1.14 with restraints that were obtained via the phenix.secondary_structure_restraints command (Adams et al., 2010). Statistics for the model were obtained using MolProbity (Chen et al., 2010) and are represented in

\section{Supplementary Table S2.}

\section{Calculation of rotation angles and vectors}

Rotation angles were calculated using UCSF Chimera with the command 'match show matrix'. The global rotation of the $18 \mathrm{~S}$ rRNA was calculated relatively to the $23 \mathrm{~S}$ rRNA by aligning all the models to the $23 \mathrm{~S}$ rRNA of a non-rotated reference structure (PDB 6SNT) (Matsuo et al., 2020). The head swivel rotation degree was calculated relatively to the $18 \mathrm{~S}$ rRNA body/platform by aligning all the model relative the body of $18 \mathrm{~S}$ rRNA from 
the reference structure (PDB 6SNT) (Matsuo et al., 2020). Vectors calculation representing a shift between the phosphate atoms of the rRNA from the model compared to the reference structure was performed using PyMol Molecular Graphics System as previously described in (Beckert et al., 2018).

\section{Figure preparation}

Figures showing biochemical experiments are fitted and plotted with GraphPad Prism 8.0. Figures showing ribosome profiling data are created using R 3.3.1.

Figures showing atomic models and electron densities were generated using either UCSF Chimera (Pettersen et al., 2004) or Chimera X (Goddard et al., 2018) and assembled with Inkscape.

\section{QUANTIFICATION AND STATISTICAL ANALYSIS}

\section{Analysis of ribosome profiling data}

The R64-1-1 S288C reference genome assembly (SacCer3) from the Saccharomyces Genome Database Project was used for yeast genome alignment. Ce10 reference genome assembly from UCSC was used for C. elegans genome alignment. $\mathrm{Hg} 19$ reference genome assembly from UCSC was used for human genome alignment. A human transcriptome file was generated to include canonical transcripts of known genes from UCSC genome browser. WT.rep1 is identical to that published previously (Wu et al., 2019). Libraries were trimmed to remove ligated 3' adapter (NNNNNNCACTCGGGCACCAAGGA), and 4 random nucleotides included $\quad$ in primer (RNNNAGATCGGAAGAGCGTCGTGTAGGGAAAGAGTGTAGATCTCGGT GGTCGC/iSP18/TTCAGACGTGTGCTCTTCCGATCTGTCCTTGGTGCCCGAGTG) were removed from the 5' end of reads. Trimmed reads longer than $15 \mathrm{nt}$ were aligned to yeast ribosomal and non-coding RNA sequences using STAR (Dobin et al., 2013) with '-outFilterMismatchNoverLmax 0.3'. Unmapped reads were then mapped to genome using the following options '--outFilterIntronMotifs RemoveNoncanonicalUnannotated -outFilterMultimapNmax 1 --outFilterMismatchNoverLmax $0.1^{\prime}$. All other analyses were performed using software custom written in Python 2.7 and R 3.3.1.

For each dataset, the offset of the A site from the 5' end of reads was calibrated using start 
codons of CDS (Schuller et al., 2017). Relative ribosome occupancies for codons or motifs were computed by taking the ratio of the ribosome density in a 3-nt window at the codon or motif of interest over the overall density in the coding sequence (excluding the first and the last $15 \mathrm{nt}$ to remove start and stop codons).

\section{Cryo-EM data analysis}

Bayesian selection using RELION software package was used to choose the cryo-EM data package (Scheres, 2012). Resolutions were calculated according to gold standard and the estimation of variation within each group of data was performed using Bayesian calculation within RELION (Scheres, 2012).

\section{AUTHOR CONTRIBUTIONS}

N.R. and S.B. performed biochemical and kinetic experiments

C.C.W. performed and analyzed the ribosome profiling experiments.

A.P. and B.B. performed cryo-EM analysis,

N.R., S.B., A.P., C.C.W., R.G., M.V.R. and D.N.W. wrote the paper.

\section{DECLARATION OF INTERESTS}

The authors declare no competing interests

\section{ACKNOWLEDGMENTS}

We thank Prof. Ralf Ficner for providing the strains for eIF1 and eIF1A purification. We thank Theresia Steiger, Tessa Hübner, Olaf Geintzer, Susanne Rieder for expert technical assistance, Paul Huter for help with data processing and Otto Berninghausen and Roland Beckmann (Gene Center, LMU, Munich) for data collection. This work has been supported by iNEXT (project number 2643 to D.N.W.), the Horizon 2020 programme of the European Union (CEITEC MU), NIH (R37GM059425 to R.G.), HHMI (R.G and C.C.W.), and grants of the Deutsche Forschungsgemeinschaft (DFG) WI3285/8-1 to D.N.W., RA 3194/1-1 to N.R. and Leibniz Prize to M.V.R.. This article reflects only the author's view and the European Commission is not responsible for any use that may be made of the 
bioRxiv preprint doi: https://doi.org/10.1101/2020.07.01.182105; this version posted July 1, 2020. The copyright holder for this preprint (which was not certified by peer review) is the author/funder, who has granted bioRxiv a license to display the preprint in perpetuity. It is made available under aCC-BY-NC-ND 4.0 International license.

information it contains. CIISB research infrastructure project LM2015043 funded by MEYS CR is gratefully acknowledged for the financial support of the measurements at the CF Cryo-electron Microscopy and Tomography CEITEC MU. 


\section{REFERENCES}

Acker, M.G., Kolitz, S.E., Mitchell, S.F., Nanda, J.S., and Lorsch, J.R. (2007). Reconstitution of Yeast Translation Initiation. Methods Enzymol 430, 111-145.

Adams, P.D., Afonine, P.V., Bunkoczi, G., Chen, V.B., Davis, I.W., Echols, N., Headd, J.J., Hung, L.W., Kapral, G.J., Grosse-Kunstleve, R.W., et al. (2010). PHENIX: a comprehensive Python-based system for macromolecular structure solution. Acta crystallographica Section D, Biological crystallography 66, 213-221.

Afonine, P.V., Grosse-Kunstleve, R.W., Echols, N., Headd, J.J., Moriarty, N.W., Mustyakimov, M., Terwilliger, T.C., Urzhumtsev, A., Zwart, P.H., and Adams, P.D. (2012). Towards automated crystallographic structure refinement with phenix.refine. Acta crystallographica Section D, Biological crystallography 68, 352-367.

Algire, M.A., Maag, D., Savio, P., Acker, M.G., Tarun, S.Z., Jr., Sachs, A.B., Asano, K., Nielsen, K.H., Olsen, D.S., Phan, L., et al. (2002). Development and characterization of a reconstituted yeast translation initiation system. RNA 8, 382-397.

Anand, M., Balar, B., Ulloque, R., Gross, S.R., and Kinzy, T.G. (2006). Domain and nucleotide dependence of the interaction between Saccharomyces cerevisiae translation elongation factors 3 and 1A. J Biol Chem 281, 32318-32326.

Anand, M., Chakraburtty, K., Marton, M.J., Hinnebusch, A.G., and Kinzy, T.G. (2003). Functional interactions between yeast translation eukaryotic elongation factor (eEF) 1A and eEF3. J Biol Chem 278, 6985-6991.

Andersen, C.B., Becker, T., Blau, M., Anand, M., Halic, M., Balar, B., Mielke, T., Boesen, T., Pedersen, J.S., Spahn, C.M., et al. (2006). Structure of eEF3 and the mechanism of transfer RNA release from the E-site. Nature 443, 663-668.

Balagopal, V., and Parker, R. (2011). Stm1 modulates translation after 80S formation in Saccharomyces cerevisiae. RNA 17, 835-842. 
Beckert, B., Turk, M., Czech, A., Berninghausen, O., Beckmann, R., Ignatova, Z., Plitzko, J.M., and Wilson, D.N. (2018). Structure of a hibernating 100S ribosome reveals an inactive conformation of the ribosomal protein S1. Nature microbiology 3, 1115-1121.

Behrmann, E., Loerke, J., Budkevich, T.V., Yamamoto, K., Schmidt, A., Penczek, P.A., Vos, M.R., Burger, J., Mielke, T., Scheerer, P., et al. (2015). Structural snapshots of actively translating human ribosomes. Cell 161, 845-857.

Belardinelli, R., Sharma, H., Caliskan, N., Cunha, C.E., Peske, F., Wintermeyer, W., and Rodnina, M.V. (2016). Choreography of molecular movements during ribosome progression along mRNA. Nat Struct Mol Biol 23, 342-348.

Ben-Shem, A., Garreau de Loubresse, N., Melnikov, S., Jenner, L., Yusupova, G., and Yusupov, M. (2011). The structure of the eukaryotic ribosome at 3.0 A resolution. Science $334,1524-1529$.

Budkevich, T., Giesebrecht, J., Altman, R.B., Munro, J.B., Mielke, T., Nierhaus, K.H., Blanchard, S.C., and Spahn, C.M. (2011). Structure and dynamics of the mammalian ribosomal pretranslocation complex. Mol Cell 44, 214-224.

Buschauer, R., Matsuo, Y., Sugiyama, T., Chen, Y.H., Alhusaini, N., Sweet, T., Ikeuchi, K., Cheng, J., Matsuki, Y., Nobuta, R., et al. (2020). The Ccr4-Not complex monitors the translating ribosome for codon optimality. Science 368 .

Chen, V.B., Arendall, W.B., 3rd, Headd, J.J., Keedy, D.A., Immormino, R.M., Kapral, G.J., Murray, L.W., Richardson, J.S., and Richardson, D.C. (2010). MolProbity: all-atom structure validation for macromolecular crystallography. Acta crystallographica Section D, Biological crystallography 66, 12-21.

Cherry, J.M., Hong, E.L., Amundsen, C., Balakrishnan, R., Binkley, G., Chan, E.T., Christie, K.R., Costanzo, M.C., Dwight, S.S., Engel, S.R., et al. (2012). Saccharomyces Genome Database: the genomics resource of budding yeast. Nucleic Acids Res 40, D700705. 
Choi, J., and Puglisi, J.D. (2017). Three tRNAs on the ribosome slow translation elongation. Proceedings of the National Academy of Sciences of the United States of America 114, 13691-13696.

Colthurst, D.R., Belfield, G.P., and Tuite, M.F. (1991). Analysis of a novel elongation factor: EF-3. Biochem Soc Trans 19, 279S.

Crowe-McAuliffe, C., Graf, M., Huter, P., Takada, H., Abdelshahid, M., Novacek, J., Murina, V., Atkinson, G.C., Hauryliuk, V., and Wilson, D.N. (2018). Structural basis for antibiotic resistance mediated by the Bacillus subtilis ABCF ATPase VmlR. Proceedings of the National Academy of Sciences of the United States of America 115, 8978-8983.

Dever, T.E., and Green, R. (2012). The elongation, termination, and recycling phases of translation in eukaryotes. Cold Spring Harb Perspect Biol 4, a013706.

Dobin, A., Davis, C.A., Schlesinger, F., Drenkow, J., Zaleski, C., Jha, S., Batut, P., Chaisson, M., and Gingeras, T.R. (2013). STAR: ultrafast universal RNA-seq aligner. Bioinformatics 29, 15-21.

dos Reis, M., Savva, R., and Wernisch, L. (2004). Solving the riddle of codon usage preferences: a test for translational selection. Nucleic Acids Res 32, 5036-5044.

Emsley, P., and Cowtan, K. (2004). Coot: model-building tools for molecular graphics. Acta crystallographica Section D, Biological crystallography 60, 2126-2132.

Firczuk, H., Kannambath, S., Pahle, J., Claydon, A., Beynon, R., Duncan, J., Westerhoff, H., Mendes, P., and McCarthy, J.E. (2013). An in vivo control map for the eukaryotic mRNA translation machinery. Mol Syst Biol 9, 635.

Fischer, N., Konevega, A.L., Wintermeyer, W., Rodnina, M.V., and Stark, H. (2010). Ribosome dynamics and tRNA movement by time-resolved electron cryomicroscopy. Nature 466, 329-333. 
Flis, J., Holm, M., Rundlet, E.J., Loerke, J., Hilal, T., Dabrowski, M., Burger, J., Mielke, T., Blanchard, S.C., Spahn, C.M.T., et al. (2018). tRNA Translocation by the Eukaryotic 80S Ribosome and the Impact of GTP Hydrolysis. Cell Rep 25, 2676-2688 e2677.

Garreau de Loubresse, N., Prokhorova, I., Holtkamp, W., Rodnina, M.V., Yusupova, G., and Yusupov, M. (2014). Structural basis for the inhibition of the eukaryotic ribosome. Nature 513, 517-522.

Goddard, T.D., Huang, C.C., Meng, E.C., Pettersen, E.F., Couch, G.S., Morris, J.H., and Ferrin, T.E. (2018). UCSF ChimeraX: Meeting modern challenges in visualization and analysis. Protein science : a publication of the Protein Society 27, 14-25.

Gomez-Lorenzo, M.G., Spahn, C.M., Agrawal, R.K., Grassucci, R.A., Penczek, P., Chakraburtty, K., Ballesta, J.P., Lavandera, J.L., Garcia-Bustos, J.F., and Frank, J. (2000). Three-dimensional cryo-electron microscopy localization of EF2 in the Saccharomyces cerevisiae 80 S ribosome at 17.5 A resolution. EMBO J 19, 2710-2718.

Grollman, A.P. (1967). Inhibitors of protein biosynthesis. II. Mode of action of anisomycin. J Biol Chem 242, 3226-3233.

Grousl, T., Ivanov, P., Malcova, I., Pompach, P., Frydlova, I., Slaba, R., Senohrabkova, L., Novakova, L., and Hasek, J. (2013). Heat shock-induced accumulation of translation elongation and termination factors precedes assembly of stress granules in S. cerevisiae. PLoS One 8, e57083.

Heuer, A., Gerovac, M., Schmidt, C., Trowitzsch, S., Preis, A., Kotter, P., Berninghausen, O., Becker, T., Beckmann, R., and Tampe, R. (2017). Structure of the 40S-ABCE1 postsplitting complex in ribosome recycling and translation initiation. Nat Struct Mol Biol 24, 453-460.

Heym, R.G., and Niessing, D. (2012). Principles of mRNA transport in yeast. Cell Mol Life Sci 69, 1843-1853. 
Ikeuchi, K., Tesina, P., Matsuo, Y., Sugiyama, T., Cheng, J., Saeki, Y., Tanaka, K., Becker, T., Beckmann, R., and Inada, T. (2019). Collided ribosomes form a unique structural interface to induce Hel2-driven quality control pathways. EMBO J 38.

Jenner, L., Starosta, A.L., Terry, D.S., Mikolajka, A., Filonava, L., Yusupov, M., Blanchard, S.C., Wilson, D.N., and Yusupova, G. (2013). Structural basis for potent inhibitory activity of the antibiotic tigecycline during protein synthesis. Proceedings of the National Academy of Sciences of the United States of America 110, 3812-3816.

Jiang, H., Lei, R., Ding, S.W., and Zhu, S. (2014). Skewer: a fast and accurate adapter trimmer for next-generation sequencing paired-end reads. BMC Bioinformatics 15, 182.

Jorgensen, R., Carr-Schmid, A., Ortiz, P.A., Kinzy, T.G., and Andersen, G.R. (2002). Purification and crystallization of the yeast elongation factor eEF2. Acta crystallographica Section D, Biological crystallography 58, 712-715.

Kamath, A., and Chakraburtty, K. (1989). Role of yeast elongation factor 3 in the elongation cycle. J Biol Chem 264, 15423-15428.

Kambampati, R., Pellegrino, C., Paiva, A., Huang, L., Mende-Mueller, L., and Chakraburtty, K. (2000). Limited proteolysis of yeast elongation factor 3. Sequence and location of the subdomains. J Biol Chem 275, 16963-16968.

Karcher, A., Schele, A., and Hopfner, K.P. (2008). X-ray structure of the complete ABC enzyme ABCE1 from Pyrococcus abyssi. J Biol Chem 283, 7962-7971.

Kasari, V., Margus, T., Atkinson, G.C., Johansson, M.J.O., and Hauryliuk, V. (2019a). Ribosome profiling analysis of eEF3-depleted Saccharomyces cerevisiae. Sci Rep 9, 3037.

Kasari, V., Pochopien, A.A., Margus, T., Murina, V., Turnbull, K., Zhou, Y., Nissan, T., Graf, M., Novacek, J., Atkinson, G.C., et al. (2019b). A role for the Saccharomyces cerevisiae ABCF protein New1 in translation termination/recycling. Nucleic Acids Res.

Kimanius, D., Forsberg, B.O., Scheres, S.H., and Lindahl, E. (2016). Accelerated cryo-EM structure determination with parallelisation using GPUs in RELION-2. eLife 5. 
Kubitschek-Barreira, P.H., Curty, N., Neves, G.W., Gil, C., and Lopes-Bezerra, L.M. (2013). Differential proteomic analysis of Aspergillus fumigatus morphotypes reveals putative drug targets. J Proteomics 78, 522-534.

Kurata, S., Shen, B., Liu, J.O., Takeuchi, N., Kaji, A., and Kaji, H. (2013). Possible steps of complete disassembly of post-termination complex by yeast eEF3 deduced from inhibition by translocation inhibitors. Nucleic Acids Res 41, 264-276.

Mateyak, M.K., Pupek, J.K., Garino, A.E., Knapp, M.C., Colmer, S.F., Kinzy, T.G., and Dunaway, S. (2018). Demonstration of translation elongation factor 3 activity from a nonfungal species, Phytophthora infestans. PLoS One 13, e0190524.

Matsuo, Y., Tesina, P., Nakajima, S., Mizuno, M., Endo, A., Buschauer, R., Cheng, J., Shounai, O., Ikeuchi, K., Saeki, Y., et al. (2020). RQT complex dissociates ribosomes collided on endogenous RQC substrate SDD1. Nat Struct Mol Biol 27, 323-332.

Milon, P., Konevega, A.L., Peske, F., Fabbretti, A., Gualerzi, C.O., and Rodnina, M.V. (2007). Transient kinetics, fluorescence, and FRET in studies of initiation of translation in bacteria. Methods in enzymology 430, 1-30.

Murina, V., Kasari, M., Takada, H., Hinnu, M., Saha, C.K., Grimshaw, J.W., Seki, T., Reith, M., Putrins, M., Tenson, T., et al. (2018). ABCF ATPases Involved in Protein Synthesis, Ribosome Assembly and Antibiotic Resistance: Structural and Functional Diversification across the Tree of Life. J Mol Biol.

Nielsen, P.R., Nietlispach, D., Mott, H.R., Callaghan, J., Bannister, A., Kouzarides, T., Murzin, A.G., Murzina, N.V., and Laue, E.D. (2002). Structure of the HP1 chromodomain bound to histone $\mathrm{H} 3$ methylated at lysine 9. Nature 416, 103-107.

Nishimura, K., Fukagawa, T., Takisawa, H., Kakimoto, T., and Kanemaki, M. (2009). An auxin-based degron system for the rapid depletion of proteins in nonplant cells. Nat Methods 6, 917-922.

Park, J.H., Wolff, E.C., and Park, M.H. (2011). Assay of deoxyhypusine hydroxylase activity. Methods Mol Biol 720, 207-216. 
Pavitt, G.D., Ramaiah, K.V., Kimball, S.R., and Hinnebusch, A.G. (1998). eIF2 independently binds two distinct eIF2B subcomplexes that catalyze and regulate guaninenucleotide exchange. Genes \& development 12, 514-526.

Pellegrino, S., Demeshkina, N., Mancera-Martinez, E., Melnikov, S., Simonetti, A., Myasnikov, A., Yusupov, M., Yusupova, G., and Hashem, Y. (2018). Structural Insights into the Role of Diphthamide on Elongation Factor 2 in mRNA Reading-Frame Maintenance. J Mol Biol 430, 2677-2687.

Percudani, R., Pavesi, A., and Ottonello, S. (1997). Transfer RNA gene redundancy and translational selection in Saccharomyces cerevisiae. J Mol Biol 268, 322-330.

Pettersen, E.F., Goddard, T.D., Huang, C.C., Couch, G.S., Greenblatt, D.M., Meng, E.C., and Ferrin, T.E. (2004). UCSF Chimera--a visualization system for exploratory research and analysis. Journal of computational chemistry 25, 1605-1612.

Phan, L., Schoenfeld, L.W., Valasek, L., Nielsen, K.H., and Hinnebusch, A.G. (2001). A subcomplex of three eIF3 subunits binds eIF1 and eIF5 and stimulates ribosome binding of mRNA and tRNA(i)Met. EMBO J 20, 2954-2965.

Rodnina, M.V. (2018). Translation in Prokaryotes. Cold Spring Harb Perspect Biol 10.

Rodnina, M.V., Semenkov, Y.P., and Wintermeyer, W. (1994). Purification of fMettRNA(fMet) by fast protein liquid chromatography. Analytical biochemistry 219, 380-381.

Sasikumar, A.N., and Kinzy, T.G. (2014). Mutations in the chromodomain-like insertion of translation elongation factor 3 compromise protein synthesis through reduced ATPase activity. J Biol Chem 289, 4853-4860.

Sattlegger, E., and Hinnebusch, A.G. (2005). Polyribosome binding by GCN1 is required for full activation of eukaryotic translation initiation factor 2 alpha\} kinase GCN2 during amino acid starvation. J Biol Chem 280, 16514-16521.

Scheres, S.H. (2012). RELION: implementation of a Bayesian approach to cryo-EM structure determination. Journal of structural biology 180, 519-530. 
Schmidt, C., Kowalinski, E., Shanmuganathan, V., Defenouillere, Q., Braunger, K., Heuer, A., Pech, M., Namane, A., Berninghausen, O., Fromont-Racine, M., et al. (2016). The cryo-EM structure of a ribosome-Ski2-Ski3-Ski8 helicase complex. Science 354, 14311433.

Schneider-Poetsch, T., Ju, J., Eyler, D.E., Dang, Y., Bhat, S., Merrick, W.C., Green, R., Shen, B., and Liu, J.O. (2010). Inhibition of eukaryotic translation elongation by cycloheximide and lactimidomycin. Nat Chem Biol 6, 209-217.

Schuler, M., Connell, S.R., Lescoute, A., Giesebrecht, J., Dabrowski, M., Schroeer, B., Mielke, T., Penczek, P.A., Westhof, E., and Spahn, C.M. (2006). Structure of the ribosomebound cricket paralysis virus IRES RNA. Nat Struct Mol Biol 13, 1092-1096.

Schuller, A.P., Wu, C.C., Dever, T.E., Buskirk, A.R., and Green, R. (2017). eIF5A Functions Globally in Translation Elongation and Termination. Mol Cell 66, 194-205 e195.

Sengupta, J., Nilsson, J., Gursky, R., Kjeldgaard, M., Nissen, P., and Frank, J. (2008). Visualization of the eEF2-80S ribosome transition-state complex by cryo-electron microscopy. J Mol Biol 382, 179-187.

Skogerson, L., and Wakatama, E. (1976). A ribosome-dependent GTPase from yeast distinct from elongation factor 2. Proceedings of the National Academy of Sciences of the United States of America 73, 73-76.

Spahn, C.M., Gomez-Lorenzo, M.G., Grassucci, R.A., Jorgensen, R., Andersen, G.R., Beckmann, R., Penczek, P.A., Ballesta, J.P., and Frank, J. (2004). Domain movements of elongation factor eEF2 and the eukaryotic $80 \mathrm{~S}$ ribosome facilitate tRNA translocation. EMBO J 23, 1008-1019.

Svidritskiy, E., Brilot, A.F., Koh, C.S., Grigorieff, N., and Korostelev, A.A. (2014). Structures of yeast $80 \mathrm{~S}$ ribosome-tRNA complexes in the rotated and nonrotated conformations. Structure 22, 1210-1218.

Szklarczyk, D., Gable, A.L., Lyon, D., Junge, A., Wyder, S., Huerta-Cepas, J., Simonovic, M., Doncheva, N.T., Morris, J.H., Bork, P., et al. (2019). STRING v11: protein-protein 
association networks with increased coverage, supporting functional discovery in genomewide experimental datasets. Nucleic Acids Res 47, D607-D613.

Taylor, D.J., Nilsson, J., Merrill, A.R., Andersen, G.R., Nissen, P., and Frank, J. (2007). Structures of modified eEF2 80S ribosome complexes reveal the role of GTP hydrolysis in translocation. EMBO J 26, 2421-2431.

Triana-Alonso, F.J., Chakraburtty, K., and Nierhaus, K.H. (1995). The elongation factor 3 unique in higher fungi and essential for protein biosynthesis is an E site factor. J Biol Chem $270,20473-20478$.

Uemura, S., Aitken, C.E., Korlach, J., Flusberg, B.A., Turner, S.W., and Puglisi, J.D. (2010). Real-time tRNA transit on single translating ribosomes at codon resolution. Nature $464,1012-1017$.

Uritani, M., and Miyazaki, M. (1988). Role of yeast peptide elongation factor 3 (EF-3) at the AA-tRNA binding step. J Biochem 104, 118-126.

Van Dyke, N., Pickering, B.F., and Van Dyke, M.W. (2009). Stm1p alters the ribosome association of eukaryotic elongation factor 3 and affects translation elongation. Nucleic Acids Res 37, 6116-6125.

Wasserman, M.R., Alejo, J.L., Altman, R.B., and Blanchard, S.C. (2016). Multiperspective smFRET reveals rate-determining late intermediates of ribosomal translocation. Nat Struct Mol Biol 23, 333-341.

Webb, K.J., Al-Hadid, Q., Zurita-Lopez, C.I., Young, B.D., Lipson, R.S., and Clarke, S.G. (2011). The ribosomal 11 protuberance in yeast is methylated on a lysine residue catalyzed by a seven-beta-strand methyltransferase. J Biol Chem 286, 18405-18413.

Weinberg, D.E., Shah, P., Eichhorn, S.W., Hussmann, J.A., Plotkin, J.B., and Bartel, D.P. (2016). Improved Ribosome-Footprint and mRNA Measurements Provide Insights into Dynamics and Regulation of Yeast Translation. Cell Rep 14, 1787-1799. 
Wohlgemuth, I., Brenner, S., Beringer, M., and Rodnina, M.V. (2008). Modulation of the rate of peptidyl transfer on the ribosome by the nature of substrates. J Biol Chem 283, 32229-32235.

Wolff, E.C., Lee, S.B., and Park, M.H. (2011). Assay of deoxyhypusine synthase activity. Methods Mol Biol 720, 195-205.

Wu, C.C., Zinshteyn, B., Wehner, K.A., and Green, R. (2019). High-Resolution Ribosome Profiling Defines Discrete Ribosome Elongation States and Translational Regulation during Cellular Stress. Mol Cell 73, 959-970 e955.

Yap, K.L., and Zhou, M.M. (2011). Structure and mechanisms of lysine methylation recognition by the chromodomain in gene transcription. Biochemistry 50, 1966-1980.

Zhang, K. (2016). Gctf: Real-time CTF determination and correction. Journal of structural biology 193, 1-12.

Zheng, S.Q., Palovcak, E., Armache, J.P., Verba, K.A., Cheng, Y., and Agard, D.A. (2017). MotionCor2: anisotropic correction of beam-induced motion for improved cryo-electron microscopy. Nat Methods 14, 331-332.

Zhou, J., Lancaster, L., Donohue, J.P., and Noller, H.F. (2014). How the ribosome hands the A-site tRNA to the P site during EF-G-catalyzed translocation. Science 345, 11881191.

Zivanov, J., Nakane, T., Forsberg, B.O., Kimanius, D., Hagen, W.J., Lindahl, E., and Scheres, S.H. (2018). New tools for automated high-resolution cryo-EM structure determination in RELION-3. eLife 7. 